\title{
Clinical exercise testing with reference to lung diseases: indications, standardization and interpretation strategies
}

\author{
ERS Task Force on Standardization of Clinical Exercise Testing \\ Members of the Task Force: J. Roca and B.J. Whipp (co-chairmen), A.G.N. Agustí, S.D. Anderson, \\ R. Casaburi, J.E. Cotes, C.F. Donner, M. Estenne, H. Folgering, T.W. Higenbottam, K.J. Killian, \\ P. Palange, A. Patessio, C. Prefaut, R. Sergysels, P.D. Wagner and I. Weisman
}

\section{CONTENTS}

\begin{tabular}{|c|c|}
\hline es to exercise in lung disease. & \\
\hline nary gas & \\
\hline tion $\ldots \ldots \ldots \ldots \ldots \ldots \ldots$ & \\
\hline al blood gases ..... & \\
\hline rascular response & \\
\hline to exercise $\ldots \ldots \ldots \ldots . . . . .$. & \\
\hline ndications ... & \\
\hline$\Delta$ & \\
\hline hronic lung diseases. & \\
\hline rative assessment & \\
\hline 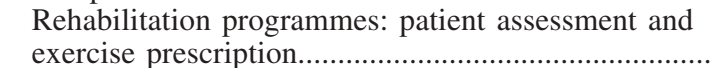 & \\
\hline ability.... & \\
\hline t-lung transplantat & \\
\hline & \\
\hline & \\
\hline
\end{tabular}

Other tests:

Constant work rate exercise testing................... 2669

Assessment of exercise-induced bronchoconstriction 2670

Equipment and quality-control programme........... 2671

Choice of ergometers......................................... 2671

Gas exchange measurements............................... 2672

The lactate threshold............................................ 2673

Exercise testing measurement systems.................. 2673

Quality control programme................................. 2675

Personnel and testing procedures.......................... 2676

Personnel qualifications........................................ 2676

Patient preparation............................................ 2676

Safety precautions............................................. 2677

Interpretative strategies..................................... 2678

General guidelines........................................... 2678

Reference values............................................. 2680

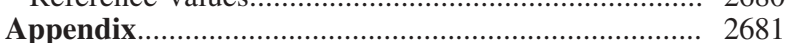

Cardiopulmonary exercise testing (CPET) is a unique tool to assess the limits and mechanisms of exercise tolerance. It also provides indices of the functional reserves of the organ systems involved in the exercise response, with inferences for system limitation at peak exercise. Moreover, CPET is useful for establishing the profiles and adequacy of the responses of the systems at submaximal exercise. The present document is essentially focused on clinical problems commonly faced in the study of patients with pulmonary diseases. Physiological changes of the respiratory system during exercise, however, should only be considered as part of a co-ordinated sequence of oxygen and carbon dioxide transfer processes between the atmosphere and the mitochondria to meet the increased energy demand of the skeletal muscle. Consequently, even in the analysis of patients with well-identified pulmonary disease, an integrative approach to CPET $[1,2]$ is required.

CPET is an area of growing interest in pulmonary medicine for three major reasons: 1) its large potential clinical applicability (see section on Indications); 2) the essentially noninvasive nature of the testing; and 3) provision of information that cannot be obtained through conventional lung function testing performed at rest [39]. During the past few years, two factors have contributed to the current level of interest in CPET in pulmonary medicine. First, substantial progress has been made in clarifying fundamental concepts of exercise physiology (e.g., factors limiting maximal oxygen uptake, lactate threshold) which have historically been the focus of controversy. Secondly, major technological improvements have facilitated data collection, subject monitoring during the test and subsequent formatting and analysis of the results. Nowadays, CPET can be considered a primary test in the pulmonary function laboratory.

The present European Respiratory Society (ERS) position document reflects the views on the topic shared by the members of the Task Force. One of the self-imposed goals of the group was to produce a relatively readerfriendly document that combined a rigorous conceptual approach with practical utility for CPET in a clinical setting. The document can be either read as a whole, or the first section (Responses to exercise in lung disease) can be used alone, as a frame of reference to clarify specific points of the document. Definitions, abbreviations,

This position document of the European Respiratory Society was officially adopted by the ERS Executive Committee on March 1 , 1997.

The Task Force on Standardization of Clinical Exercise Testing was endorsed by the Clinical Physiology Assembly and the Clinical Assembly of the ERS.

Correspondence: J. Roca, Servei de Pneumologia, Hospital Clínic, Barcelona, Spain; or B.J. Whipp, Dept of Physiology, St. George's Hospital, London, UK. 
information content and units of the recommended variables are presented in the Appendix. Finally, as a group, the Task Force acknowledges that the introduction of SI units has represented a valuable effort not only to provide a coherent system of units, but also to ensure that units are uniform in concept and style. Unfortunately, the use of SI is still insufficiently widespread in clinical exercise testing. In order to avoid nomenclature that can be unfamiliar to many readers, we decided to use traditional units in the text but also provide the equivalences between the two systems of units in the Appendix.

\section{Responses to exercise in lung disease}

Exercise intolerance results when a subject is unable to sustain a required work rate (WR) sufficiently long for the successful completion of the task. The cause, most commonly, is an oxygen demand that exceeds the maximal oxygen conductance capability of the oxygen transport chain; the consequence is a perception of limb fatigue, breathlessness or even, in some conditions, frank pain. Subjects with lung disease often experience exercise intolerance at extremely low work rates. There are many kinds of lung disease, however, and in any one patient the structural and functional severity of the disease may range from the barely discernible to the very severe. As a result, responses to exercise in patients with lung disease do not show the tight stereotypical pattern of normal subjects.

The appropriateness of the integrated systemic responses are perhaps best studied utilizing incremental exercise testing either as a ramp or small WR increments each of short duration. This provides a convenient means of: 1) determining whether the magnitude and pattern of response of particular variables is normal with respect to other variables or to WR; 2) establishing a subject's limiting or maximum attainable value for physiological variables of interest; and 3) establishing exercise intensity domains, such as the transition between moderate and heavy intensity exercise. It is important to recognize, in this context, the difference between submaximal and maximal exercise levels. In submaximal exercise, the components of the oxygen transport pathway can provide adequate oxygen and carbon dioxide fluxes between the air and the mitochondria. Mitochondrial oxidative capacity has not been reached, symptoms are usually tolerable and muscle fatigue has not occurred, or at least may be insufficient to impair performance appreciably. At maximal exercise, symptoms have caused the patient to stop exercising. At this stage, one or more of the following possibilities exist: 1) Limits to oxygen transport have been reached and maximal oxygen consumption $\left(V^{\prime} \mathrm{O}_{2}\right.$, max $)$ attained; under such conditions, breathing $100 \%$ oxygen, for example, could increase $V^{\prime} \mathrm{O}_{2}$, max [10]; 2) Mitochondrial oxidative capacity has been reached and again the subject would be considered to be at $V^{\prime} \mathrm{O}_{2}$, max, but adding oxygen would not raise oxygen consumption $\left(V^{\prime} \mathrm{O}_{2}\right) ; 3$ ) Maximal exercise has occurred at a level that does not require maximal oxygen transport or maximal oxidative capacity and here exercise has been limited by unusually severe symptoms. Under these conditions a plateau in oxygen uptake
$\left(V^{\prime} \mathrm{O}_{2}\right.$, max $)$ has not been reached and the appropriate term is peak rather than maximal $V^{\prime} \mathrm{O}_{2}$.

\section{Pulmonary gas exchange}

Oxygen uptake. The peak $V^{\prime} \mathrm{O}_{2}$ is typically low in patients with lung disease $[2,5,11]$. The increment in $V^{\prime} \mathrm{O}_{2}$ in response to incremental or steady state exercise, however, occurs in a manner that often cannot be separated from that of normal subjects. That is, the response is linear with a slope $\left(\Delta V^{\prime} \mathrm{O}_{2} /\right.$ change in work rate $\left.(\Delta \mathrm{WR})\right)$ for cycle ergometry of approximately $10 \mathrm{~mL} \cdot \mathrm{min}^{-1} \cdot \mathrm{W}^{-1}$ (i.e. within the $95 \%$ confidence limits $(95 \% \mathrm{CI})$ of 8.5 and 11.5 for normal subjects) [12]. Yet the oxygen cost of breathing per unit ventilation is increased in both chronic obstructive pulmonary disease (COPD) and interstitial lung disease (ILD). In COPD, airway obstruction requires more effort to move a given volume of air, while in ILD increased elastic recoil requires more inspiratory muscle activity. In mild disease these additional costs can be quite small; in severe disease the expected higher $V^{\prime} \mathrm{O}_{2}$ from the extra cost of breathing may be offset by the slower oxygen transport and utilization kinetics (patients also tend to be sedentary) [13].

Carbon dioxide output $\left(\mathrm{V}^{\prime} \mathrm{CO}_{2}\right)$. Because the tissue capacitance for carbon dioxide is markedly greater than for oxygen, pulmonary carbon dioxide output will initially not rise as fast as pulmonary uptake of oxygen, despite similar rates of metabolic exchange. For moderate constant work-rate exercise in healthy young subjects, this effect prolongs the 30-40 s time constant of metabolic $V^{\prime} \mathrm{CO}_{2}$ from the start of exercise (considered to be closely equivalent to that of pulmonary oxygen uptake) to a value of 50-60 s for pulmonary carbon dioxide exchange $[14,15]$. This transient storage effect is exacerbated in patients with COPD who have areas of high alveolar ventilation/perfusion $\left(V^{\prime} \mathrm{A} / Q^{\prime}\right)$ ratios. Commonly, these regions receive as much as $50 \%$ of the alveolar ventilation but only $5 \%$ or less of the cardiac output [16-18]. As a result, the bulk of the $V^{\prime} \mathrm{CO}_{2}$ comes from relatively poorly ventilated areas of the lung. This, in addition to any overall slowing of the pulmonary blood flow, can retard the kinetics of carbon dioxide elimination, which is also seen in those pulmonary vascular disease (PVD) patients with high $V^{\prime} \mathrm{A} / Q^{\prime}$ regions.

\section{Ventilation}

The appropriateness of the ventilatory response to exercise depends not on the actual level of ventilation achieved but rather on the extent to which it subserves its pulmonary gas exchange and acid-base regulatory requirements. The ventilatory response to exercise, however, is influenced not only by metabolic rate (most closely for carbon dioxide) but also by the regulated or "set point" level of arterial carbon dioxide tension $\left(\mathrm{Pa}_{\mathrm{a}} \mathrm{CO}_{2}\right)$ and the physiological dead-space fraction of the breath. Quantitatively, these are represented by the following relationship:

$$
V^{\prime} \mathrm{E}=\left[863 \cdot V^{\prime} \mathrm{CO}_{2}\right] /\left[P \mathrm{~A}, \mathrm{CO}_{2} \cdot(1-V \mathrm{D} / V \mathrm{~T})\right]
$$


where 863 is the constant which corrects for the different conditions of reporting the gas volumes (for a body temperature of $37^{\circ} \mathrm{C}$ ) and also the transformation of fractional concentration to partial pressure; $V^{\prime} \mathrm{E}$ is minute ventilation in litres per minute; $P \mathrm{~A}, \mathrm{CO}_{2}$ indicates alveolar carbon dioxide tension in millimetres of mercury (usually taken to be equivalent to $P \mathrm{a}, \mathrm{CO}_{2}$ ); and, $V \mathrm{D} /$ $V \mathrm{~T}$ is the dead space (VD) expressed as a fraction of the tidal volume $(V \mathrm{~T})$.

Minute ventilation ( $\mathrm{V}^{\prime} E$ ). Lung disease typically increases both ventilation at rest and the ventilatory requirements for a given level of exercise $[2,19,20]$. The high work of breathing may also lead to respiratory muscle fatigue. This may produce limiting symptoms, although shortness of breath cannot necessarily be equated to respiratory muscle fatigue. Resting ventilation is usually greater than normal in patients with COPD, ILD and pulmonary vascular disease (PVD). This is indirectly due to the presence of $V^{\prime} \mathrm{A} / Q^{\prime}$ inequality produced by the classical pathological abnormalities of emphysema and chronic bronchitis in COPD and by fibrosis in ILD [21]. Thus, an abnormal level of ventilation is required to maintain normal $P \mathrm{a}, \mathrm{CO}_{2}$, even at rest, when there is $V^{\prime} \mathrm{A} / Q^{\prime}$ mismatching. This is especially so in emphysematous patients who often show not only the expected higher ventilation needed for eucapnia as a result of high $V^{\prime} \mathrm{A} / Q^{\prime}$ areas, but even higher ventilation as evidenced by low $\mathrm{Pa}, \mathrm{CO}_{2}$ (i.e. $<40 \mathrm{mmHg}(5.3$ $\mathrm{kPa})$ ). What drives them to hyperventilate remains obscure. Other patients with COPD have carbon dioxide retention even at rest. The carbon dioxide retention, of course, does not necessarily indicate that the $V^{\prime} E$ is less than normal, only that the normal defence of $P \mathrm{a}, \mathrm{CO}_{2}$ has failed. In ILD and pulmonary vascular disease (PVD) there is often considerable hyperventilation even at rest [22-24], with $P \mathrm{a}_{1} \mathrm{CO}_{2}$ in the $30-35 \mathrm{mmHg}(4.0-4.7 \mathrm{kPa})$ range. This can occur even when $\mathrm{Pa}_{\mathrm{a}} \mathrm{O}_{2}$ is at or above $60 \mathrm{mmHg}(8.0 \mathrm{kPa})$.

Breathing pattern. In patients with chronic lung diseases, the $V \mathrm{~T}$ tends to be lower and the respiratory frequency $(f \mathrm{R})$ higher for a given level of $V^{\prime} \mathrm{E}[2,25]$. A strong linear relationship between peak exercise $V \mathrm{~T}$ and vital capacity (VC) has been shown both in patients with COPD and idiopathic pulmonary fibrosis (IPF), suggesting that the differences in peak $V$ T are mainly due to abnormal respiratory mechanics. However, determining $V \mathrm{~T}$ at submaximal exercise is also useful. During exercise, normal human subjects increase $f \mathrm{R}$ by decreasing inspiratory time $(t \mathrm{I})$ fractionally less than expiratory time $(t \mathrm{E})$. As a result, the ratio of $t \mathrm{I}$ to total breath duration (ttot) (the inspiratory duty cycle $(t \mathrm{I} / t \mathrm{tot})$ ) increases in normal humans from $0.35-0.40$ at rest to $0.50-0.55$ during exercise. Patients with ILD show a similar type of response. In contrast, patients with severe COPD often show no increase in $t \mathrm{t} / t$ tot with exercise, thus preserving greater time for expiration.

Pulmonary mechanics. Does ventilation during exercise reach mechanical limits in patients with lung disease? Certainly, flow rates (inspiratory and/or expiratory) can be shown to reach the envelope of the resting maximal flow volume curve, particularly during expiration in COPD $[26,27]$ and even in some elderly normal subjects who are physically fit [28]. As this may be expected to result in abnormal respiratory sensations, this may contribute to exercise limitation. However, unless flow limitation is occurring through a substantial majority of expiration rather than over a small part of the breath, there is no capacity to increase lung volume (to advantage airflow) and $f \mathrm{R}$ cannot be increased without compromising $V \mathrm{~T}$, it may well be that total ventilation is not at a mechanically defined upper limit, even if a portion of respiratory flow is. Patients with COPD adopt two strategies during exercise to increase $V^{\prime} \mathrm{E}$ when there is expiratory flow limitation: 1) end-expiratory lung volume (EELV) increases [26, 27], in contrast to normal humans who show a fall in EELV during exercise [29], or in patients with ILD, who do not change EELV significantly during exercise $[29,30]$; and 2) inspiratory flow rate increases with decreased inspiratory time (i.e., allowing more time for expiration). Traditionally, "ventilatory limitation" during exercise has been expressed by comparing exercise ventilation to the resting maximal voluntary ventilation (MVV) as an estimate of ventilatory capacity. However, while convenient, MVV is not an ideal yardstick of whether a limit to ventilation has occurred during exercise. The MVV manoeuvre is performed over only $12-15 \mathrm{~s}$ at $V \mathrm{~T}$ and $f \mathrm{R}$ values that usually differ from those adopted naturally during exercise, and also at different end-expiratory/inspiratory lung volumes. Another approach to assessing whether ventilation during exercise has attained its limiting value has been to add carbon dioxide to the inspired gas during exercise [17, 31]. If ventilation increases in response to the added carbon dioxide, then it can be concluded that ventilation in the absence of carbon dioxide was not mechanically limited. However, failure to increase ventilation in response to added carbon dioxide or to added external dead space, for example, could be due either to mechanical limitation or to insufficient chemoreceptor stimulation to increase ventilation in the face of other ventilatory constraints. A further approach has been to unload the respiratory system, by breathing $21 \%$ oxygen in helium rather than in air. This usually increases ventilation during maximal exercise in both COPD patients [17, 32] and normal subjects [33, 34]. However, one is now dealing with a mechanically altered respiratory system with a reduced work of breathing per unit ventilation. Such a result is not necessarily evidence that ventilation while respiring room air was mechanically limited.

Respiratory muscle function. There are several reasons to suggest that respiratory muscle function during exercise can be a limiting factor in patients with lung disease. Most prominent among them are: 1) the load to the respiratory muscles is increased both in COPD (airflow obstruction) and ILD (increased lung elastance); 2) in COPD the diaphragm is at a mechanical disadvantage due to dynamic hyperinflation; and 3) hypoxaemia during exercise may render respiratory muscles prone to fatigue.

Bronchomotor tone response to exercise. The normal bronchomotor response to exercise is a mild degree of bronchodilation. Patients with exercise-induced asthma 
and some patients with COPD may show exercise-induced bronchoconstriction, but this is most commonly a postexercise phenomenon. Exercise testing is, by definition, useful in investigating the mechanisms of exercise-induced bronchoconstriction.

\section{Arterial blood gases}

Many patients with COPD, ILD or PVD are hypoxaemic at rest breathing room air at sea level. That is, their $P \mathrm{a}, \mathrm{O}_{2}$ is below the lower reference limit, i.e 80 $\mathrm{mmHg}(10.6 \mathrm{kPa})$. There is, however, great variability, with $\mathrm{Pa}, \mathrm{O}_{2}$ being within normal limits for age or as low as $30-40 \mathrm{mmHg}(4.0-5.3 \mathrm{kPa})$. In COPD, most of the patients show mild-to-moderate hypoxaemia 60-70 mmHg (8.0-9.3 kPa). Those COPD patients who retain carbon dioxide at rest are generally more hypoxaemic, with $\mathrm{Pa}, \mathrm{O}_{2}$ often being in the $40-60 \mathrm{mmHg}(5.3-8.0$ $\mathrm{kPa}$ ) range. A similar broad range of $\mathrm{Pa}, \mathrm{O}_{2}$ is seen in ILD and PVD. $P \mathrm{a}_{\mathrm{a}} \mathrm{CO}_{2}$ is usually normal or slightly reduced in those COPD patients with $\mathrm{Pa}_{\mathrm{a}} \mathrm{O}_{2}$ in the 60-70 $\mathrm{mmHg}(8.0-9.3 \mathrm{kPa})$ range. Carbon dioxide retainers, of course, have an elevated $\mathrm{Pa}, \mathrm{CO}_{2}$, usually in the range $45-55 \mathrm{mmHg}(6.0-7.3 \mathrm{kPa})$, but again extremes exist with values as high as $70-80 \mathrm{mmHg}(9.3-10.6 \mathrm{kPa})$ occasionally seen, even in stable out-patients. In ILD, $P \mathrm{a}, \mathrm{CO}_{2}$ is typically $30-35 \mathrm{mmHg}(4.0-4.7 \mathrm{kPa})$, as discussed previously. This is also true in PVD. From these general pairings of partial pressure of oxygen $\left(\mathrm{PO}_{2}\right)$ and partial pressure of carbon dioxide $\left(\mathrm{PCO}_{2}\right)$, the alveolar gas equation can be used to deduce the alveolar-arterial oxygen difference $\left(\mathrm{DA}-\mathrm{a}, \mathrm{O}_{2}\right)$ :

$$
\begin{gathered}
\text { DA-a }, \mathrm{O}_{2}=\left(P \mathrm{I}, \mathrm{O}_{2}-P \mathrm{a}, \mathrm{CO}_{2} / \mathrm{RER}+\right. \\
\left.\left(P \mathrm{a}, \mathrm{CO}_{2} \cdot F \mathrm{I}, \mathrm{O}_{2} \cdot(1-\mathrm{RER}) / \mathrm{RER}\right)\right)-P \mathrm{a}, \mathrm{O}_{2}
\end{gathered}
$$

where RER is the respiratory exchange ratio (RER= $\left.V^{\prime} \mathrm{CO}_{2} / V^{\prime} \mathrm{O}_{2}\right) P \mathrm{I}, \mathrm{O}_{2}$ is the inspiratory oxygen pressure and $F \mathrm{I}, \mathrm{O}_{2}$ is the inspiratory oxygen fraction. This equation is derived from mass balance considerations of oxygen and carbon dioxide exchange between the air and the alveolar gas and assumes constancy of lung nitrogen stores. Thus, even if $V^{\prime} \mathrm{O}_{2}, V^{\prime} \mathrm{CO}_{2}$ and $V^{\prime} \mathrm{E}$ are changing, the DA-a, $\mathrm{O}_{2}$ can be validly computed if there is nitrogen balance between inspiration and expiration. It is important to recognize that the absolute blood-gas values and the DA-a, $\mathrm{O}_{2}$ yield different, but complementary information. The DA-a, $\mathrm{O}_{2}$ reflects primarily pulmonary defects in gas exchange caused by $V^{\prime} \mathrm{A} / Q^{\prime}$ mismatching, diffusion limitation and shunt either alone or in combination. It can, however, be modified by changes in cardiac output or ventilation even in the absence of change in $V^{\prime} \mathrm{A} / Q^{\prime}$ distribution. The typical response to exercise in COPD is a small rise in $P \mathrm{a}_{1} \mathrm{CO}_{2}$ and a similar, or even greater, fall in $\mathrm{Pa}, \mathrm{O}_{2}$. However, $\mathrm{Pa}, \mathrm{O}_{2}$ frequently does not fall, and may even increase in some subjects. Studies using the multiple inert gas elimination technique show that $V^{\prime} \mathrm{A} / Q^{\prime}$ mismatch is usually unaltered from that at rest in COPD, that shunts do not develop, and that diffusion limitation also does not occur $[16,35]$. This is particularly so when COPD is severe. In milder disease, there is evidence that small improvements in $V^{\prime} \mathrm{A} / Q^{\prime}$ relationships may occur on exercise [18]. The blood-gas changes on exercise are mostly the consequence of how alveolar ventilation increases compared to $V^{\prime} \mathrm{O}_{2}$ and $V^{\prime} \mathrm{CO}_{2}$, with secondary effects from the fall in mixed venous $\mathrm{PO}_{2}$. However, it is not infrequently observed that when the patient with COPD is encouraged to maximal effort, sudden hypoxaemia and hypercapnia can develop. In ILD the bloodgas changes with exercise are usually more typical and substantial even at moderate effort. While $\mathrm{Pa}_{\mathrm{a}} \mathrm{CO}_{2}$ is generally unaffected, $\mathrm{Pa}, \mathrm{O}_{2}$ falls in almost all patients with diffusion limitation being contributory [23]. It is remarkable that diffusion limitation of oxygen exchange can occur even at a cardiac output of less than $10 \mathrm{~L} \cdot \mathrm{min}^{-1}$ during exercise in ILD. However, as with COPD, the amount of $V^{\prime} \mathrm{A} / Q^{\prime}$ mismatching and shunt appear not to change with exercise [23]. In PVD, $\mathrm{Pa}_{\mathrm{a}} \mathrm{O}_{2}$ usually falls by several millimetres of mercury, $\mathrm{Pa}, \mathrm{CO}_{2}$ also falls by perhaps $3-5 \mathrm{mmHg}(0.40-0.67 \mathrm{kPa})$, and the $\mathrm{DA}-\mathrm{a}, \mathrm{O}_{2}$, thus, increases. As with ILD, this is found to be largely due to the large fall in venous $P_{\mathrm{O}_{2}}$ rather than a systematic change in $V^{\prime} \mathrm{A} / Q^{\prime}$ relationships [23], and diffusion limitation may also develop.

\section{Cardiovascular response}

It should be remembered that patients with lung disease are subject to the same general cardiovascular disorders as anyone else. Those with COPD in particular share risk factors for ischaemic heart disease due to cigarette smoking, age and inactivity, and in addition they are generally hypoxaemic. However, throughout the following discussion, ischaemic heart disease is assumed not to be present. Also, it is well known that eventually many patients with either COPD or ILD will develop cor pulmonale with right heart failure from their lung disease. This will also happen in PVD. While the pulmonary circulation shows evidence of abnormality well before frank right heart failure develops, the following discussion is limited to those patients who have not yet reached the stage of clinical right heart failure. Typically cardiac output increases per unit increase in metabolic rate even in patients with very severe lung disease [16, 36], except in some with PVD. This is true even if cardiac output is somewhat reduced at rest. However, at peak exercise in these forms of lung disease, cardiac output is about $50 \%$ (less in PVD) of what a normal older subject could achieve at peak exercise [18, 36]. There are two possible explanations. First, the control of cardiac output during exercise in lung disease may remain so tight that, despite the capacity for a higher cardiac output, it remains regulated to match the level of $V^{\prime} \mathrm{O}_{2}$ achieved. The second is that despite absence of overt heart failure, cardiac function is indeed compromised and a higher cardiac output could not be achieved. Pulmonary hypertension is often evident even at rest, and usually worsens during exercise. The increase in pressure per unit increase in cardiac output is sometimes three times greater in the patient groups than in normal subjects. In normal subjects, although pulmonary artery pressure normally rises during exercise, pulmonary vascular resistance normally falls due to a combination of vascular recruitment and distension in the lungs. In ILD, PVD and severe COPD on the other hand, vascular resistance remains constant or may even rise $[18$, 
$23,24]$. The reason is vascular destruction or obstruction, which is known to occur in these diseases, and hypoxic vasoconstriction. Eventually, as the diseases progress, the right heart will hypertrophy and ultimately fail, and clinically significant cor pulmonale will be present.

Despite the marked increase in vascular resistance, it is remarkable that even in advanced disease the heart can pump in an essentially normal manner as a function of filling pressure if heart failure is not present. Cardiac frequency $(f \mathrm{C})$ at a given $V^{\prime} \mathrm{O}_{2}$ is higher than normal in subjects with these lung diseases. This implies that stroke volume must be lower. Whether this is simply an extension of the phenomenon in health, whereby unfit subjects have a higher $f \mathrm{C}$ than fit persons at the submaximal $V^{\prime} \mathrm{O}_{2}$, is unclear. The oxygen pulse $\left(V^{\prime} \mathrm{O}_{2}\right.$ per heart beat) is an index that is in common current use (see [2] pages 65-66 and 119-120 for discussion) because: 1) of its noninvasive nature; and 2) it is quantitatively equivalent to the product of stroke volume and arterial minus mixed venous oxygen difference. This will be lower in lung disease if $f \mathrm{C}$ is higher. However, despite the $f \mathrm{C}$ being higher at the same $V^{\prime} \mathrm{O}_{2}$ than in health, $f \mathrm{C}$ at peak $V^{\prime} \mathrm{O}_{2}$ is almost always less than predicted. As the $V^{\prime} \mathrm{O}_{2}$-cardiac output relationship is generally normal, the arteriovenous oxygen concentration difference will also be normal for a given $V^{\prime} \mathrm{O}_{2}$. However, both arterial and mixed venous $\mathrm{PO}_{2}$ levels are reduced due to the hypoxaemia of lung disease. Interestingly, mixed venous $\mathrm{PO}_{2}$ at peak exercise in health is about the same as in lung diseases, despite the much higher peak $V^{\prime} \mathrm{O}_{2}$ in health.

\section{Limits to exercise}

At levels of effort below peak or maximal, patients with lung disease may have a total ventilation and cardiac output increase, at a given $V^{\prime} \mathrm{O}_{2}$, at least as much as in health even if respiratory muscle and cardiac work and the patient's symptoms are greater. The physiological basis for reduction in peak or maximal $V^{\prime} \mathrm{O}_{2}$ is of major interest. The reduction in peak $V^{\prime} \mathrm{O}_{2}$ in COPD patients, for example, sometimes cannot be accounted for simply by "ventilatory limitation"; in fact, leg fatigue is a commonly reported end-exercise symptom in these patients [17]. Although they may not have been able to achieve higher ventilation breathing room air because of their lung disease, there are two additional factors of considerable quantitative importance contributing to the low peak $V^{\prime} \mathrm{O}_{2}$. Firstly, the cardiac output at peak exercise is about $50 \%$ of the value that a normal agematched subject would achieve at maximal effort. Whether this represents cardiac dysfunction or, as stated, earlier manifestation of the tight regulation between $V^{\prime} \mathrm{O}_{2}$ and cardiac output, is uncertain. However, oxygen transport would presumably have been greater had cardiac output reached normal maximal values and (subject to not having reached mitochondrial oxidative limitation) the same would have been true of maximal $V^{\prime} \mathrm{O}_{2}$. A second factor that might decrease peak $V^{\prime} \mathrm{O}_{2}$ is a reduction in muscle oxygen conductance [37], resulting from inhomogeneity between local muscle oxygen and blood flow or to reduced diffusional conductance, possibly the result of myopathic capillary rarification.
Had the muscle conductance been normal, peak $V^{\prime} \mathrm{O}_{2}$ could then have been greater even at the same cardiac output and $\mathrm{Pa}, \mathrm{O}_{2}$. It is also unclear whether simple lack of fitness or additional pathological factors are responsible. Of course, if $V^{\prime} \mathrm{O}_{2}$ could be improved by such therapeutic gains, $V^{\prime} \mathrm{CO}_{2}$ would rise accordingly and provide an added burden to ventilation that might still prevent the anticipated gain in $V^{\prime} \mathrm{O}_{2}$,max. Recognizing the potential capacity for factors other than ventilation to limit exercise in lung disease is important in attempting to correct the primary lung problem, e.g. by transplantation. For example, the poor exercise responses after organ transplantation in cardiopulmonary diseases are likely to be due, at least in part, to unrecognized and untreated defects in skeletal muscle blood flow and oxygen transport.

\section{Indications}

There is a range of indications for CPET, as outlined in table 1. It is useful, for example, in the diagnosis of a range of disease conditions, such as: 1) exercise-induced asthma; 2) cardiac ischaemia; 3) foramen ovale patency with development of right-to-left shunt during exercise [2]; and 4) McArdle's syndrome [38]. In addition, CPET provides information on dysfunction, monitoring and prognostic value in a wide range of conditions (table $1 ;[5])$.

However, an adequate identification of the clinical problem requiring study should be considered a necessary prelude to CPET, as should an appropriate assessment of the patient by: 1) medical history; 2) physical examination; 3) chest radiograph; 4) pulmonary function testing; and 5) electrocardiogram (ECG). The clinical problem that prompts the CPET and the specific aims of the test (i.e. assessment of exercise tolerance, analysis of pulmonary gas exchange during exercise,

Table 1. - Indications for cardiopulmonary exercise testing with reference to lung diseases

Assessing exercise tolerance and potential limiting factors Identification of abnormal limitation of exercise intolerance, and discrimination among causes of exercise tolerance

Differentiation between dyspnoea of cardiac or pulmonary origin

Evaluation of unexplained dyspnoea when initial pulmonary lung function impairment does not provide conclusive results Assessing impairment in chronic lung diseases

Interstitial lung diseases

Chronic obstructive pulmonary disease

Marked hypoxaemia during exercise

Occult cardiac disease

Abnormal breathing pattern during exercise

Chronic pulmonary vascular occlusion (controversial)

Cystic fibrosis

Preoperative assessment

Major abdominal surgery, especially in elderly patients

Lung cancer resectional surgery

Resectional surgery in pulmonary emphysema

Diagnosis of exercise-induced asthma

Rehabilitation programmes: patient assessment and exercise prescription

Assessment of impairment-disability

Lung, heart-lung transplantation

Modified from [5]. 


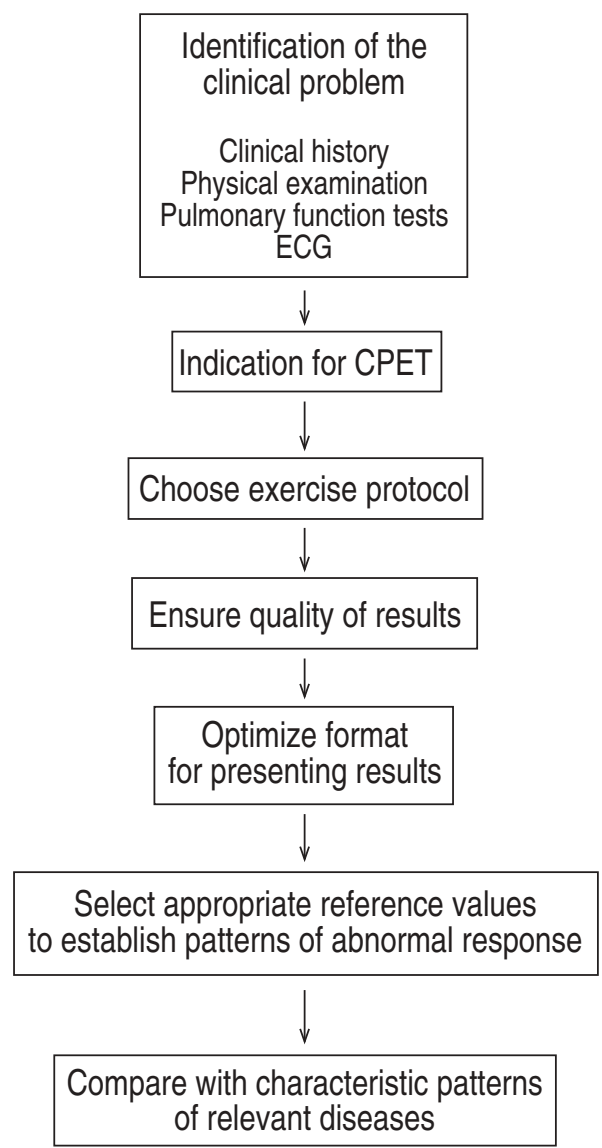

Fig. 1. - Logical strategy to approach cardiopulmonary exercise testing (CPET) in a clinical setting. ECG: electrocardiogram.

etc.) determine both the type of exercise protocol to be used and the variables to be considered in the interpretation of the test (fig. 1). These are described in detail below (see sections on exercise protocols and interpretation strategies).

\section{Assessing exercise tolerance and potential limiting fac- tors}

Exercise performance and $V^{\prime} \mathrm{O}_{2}$ peak cannot be validly predicted from resting cardiopulmonary testing. This is especially true in patients in whom exercise is limited by lung function [3-9]. This is not only because exertional dyspnoea is not well predicted from pulmonary function, but also because many patients with lung disease stop exercising owing to fatigue, chest pain and leg discomfort rather than dyspnoea [3]. For these reasons and because measures of health-related quality of life correlate better with exercise tolerance than with either spirometry or oxygenation [9], CPET is a useful tool in the integrated evaluation of common problems such as unexplained dyspnoea on exertion and limitation of exercise tolerance $[39,40]$. Appropriate use of CPET allows the investigator: 1) to quantify the degree of abnormal limitation and to discriminate among causes of exercise intolerance; 2) to differentiate between dyspnoea of cardiac or pulmonary origin when respiratory and cardiac diseases coexist; and 3) to analyse unexplained dyspnoea when initial pulmonary function impairment does not provide conclusive results [5, 40].

\section{Assessment of impairment in chronic lung diseases}

Comprehensive CPET can be extremely useful in different phases of the decision-making process in chronic lung diseases.

Interstitial lung diseases. Assessment of pulmonary gas exchange during exercise [23, 41-43] in the clinical evaluation of ILD is fundamental to early diagnosis, staging of dysfunction and monitoring of therapy. Moreover, recent data in a rather small group of subjects [44] seem to suggest that CPET might also have a prognostic value in idiopathic ILD.

Chronic obstructive pulmonary disease. Use of CPET to assess the severity of dysfunction in COPD can be valuable in the detection of situations that may prompt therapeutic decisions to ameliorate the consequences of the disease such as: 1) marked hypoxaemia during exercise (needs for supplemental oxygen can be assessed directly; 2) occult cardiac disease (initiate appropriate therapeutic regime); or 3) inefficient breathing patterns during exercise (initiate rehabilitation programme?). Use of CPET in prospective trials may also provide information in the evaluation of new experimental therapeutic approaches such as resectional surgery in pulmonary emphysema [45, 46].

Chronic pulmonary vascular occlusion. It has been shown that $V^{\prime} \mathrm{O}_{2}$ peak is closely correlated to the amount of functional vascular bed in chronic pulmonary vascular occlusion [22, 47]. However, exercise testing in such patients carries a significant mortality risk and should not be performed when there is a history of arrhythmias or syncope, or clinical signs of right heart failure. Furthermore, the power output attainable during exercise in patients with primary pulmonary hypertension correlates well with haemodynamic variables measured at rest [48-50]. Consequently, indications of CPET in such patients should be carefully established on an individual basis.

Cystic fibrosis. There is evidence to support the use of CPET as a tool in the prognosis and management of patients with cystic fibrosis [51].

\section{Preoperative assessment}

Major abdominal surgery in elderly patients. While mortality remains high in elderly patients undergoing major abdominal surgery, chronological age is a poor guide to physiological status. It has been suggested that reduction of cardiopulmonary reserve during exercise can provide a reasonable prediction of mortality following major surgery $[52,53]$. It has been suggested that there is a need for a system for grading operative risk that includes evidence of abnormal cardiopulmonary function based on CPET.

Preoperative assessment of lung cancer resectional surgery. Information on predicted postoperative lung function: 1) helps to modulate the amount of lung parenchyma to be resected; and 2) determines the type of 
preoperative strategy needed to prevent postsurgical complications. Resting pulmonary function tests are considered adequate to evaluate patients with low risk (forced expiratory volume in one second (FEV1) $>2 \mathrm{~L}$ and transfer factor of the lung for carbon monoxide $(T \mathrm{~L}, \mathrm{CO})$ within the reference limits) of postsurgical complications [54-59]. However, CPET plays a pivotal role in the evaluation of patients with moderate to high risk $[57,58,60,61]$.

Preoperative assessment of lung-reduction surgery. Lung-reduction surgery $[45,46,62]$ is the newest therapeutic option for patients with emphysema, but still requires further evaluation. As indicated above, CPET plays a key role in this type of surgery, both in the selection of potential candidates and to establish the programme of physical rehabilitation before and after surgical intervention.

\section{Rehabilitation programmes: patient assessment and exer- cise prescription}

Exercise therapy should be part of rehabilitation programmes that aim to improve both quality of life and physiological status in patients with COPD and other forms of chronic lung disease [3, 63-72]. CPET should always play a central role in the assessment of candidates before the rehabilitation programme and in the subsequent modulation of the exercise prescription, whereas simpler tests (i.e. 6 min walk) are useful for monitoring during the rehabilitation programme.

While it is accepted that physical training can produce physiological improvements in patients with moderate to severe COPD - such as: 1) increase in exercise tolerance $\left(V^{\prime} \mathrm{O}_{2}\right.$ peak) $\left.[71] ; 2\right)$ decrease in ventilatory requirements [73]; and 3) faster oxygen kinetics [74] controversy still remains regarding the type of training programme that is most appropriate [63-65].

\section{Assessment of impairment-disability}

It is now well accepted that CPET provides different and relevant information in impairment-disability evaluation [5-7], compared to resting cardiopulmonary measurements [75]. Consequently, CPET constitutes a key tool in this area. Clarification of this field should be greatly facilitated with the adoption of the conceptual framework proposed by the World Health Organization (WHO) [76]. According to the WHO, the illness-related phenomena should be classified in three different categories: 1) impairment, used to describe loss of function; 2) disability, corresponds to the resulting reduction in exercise capacity; and 3) handicap, the total effect of the illness on the subject's social life.

\section{Lung, heart-lung transplantation}

Lung and heart-lung transplantation are now accepted as viable therapeutic options for patients with end-stage vascular and parenchymal lung diseases. CPET is a useful tool in the preoperative phase of the evaluation of the patient's impairment. CPET may also contribute to the evaluation of disease progression [77], the appropriate timing for surgical intervention [78, 79], and can be used to guide preoperative rehabilitation [48, 8083]. As yet, however, there are no recommendations as to how indices of exercise performance should be used in the decision-making process of when a patient should be selected for a lung transplant. In contrast, such recommendations do exist, and have been successfully applied, in candidates for cardiac transplantation; survival has been shown to be well correlated with $V^{\prime} \mathrm{O}_{2}$ peak $[78,79]$.

Several studies have shown that pulmonary and cardiac function are typically satisfactory in patients with adequate allograft tolerance. The majority of these patients report considerable improvement in functional outcome and life satisfaction, often with resumption of a normal life style. The available post-transplant data demonstrate that virtually all recipients have persistent exercise impairment, regardless of the underlying disease or type of transplant procedure [83]. This is primarily attributed to skeletal muscle dysfunction [83-85]. In the postoperative phase, CPET is useful in the assessment of limitation of exercise tolerance, discriminating among its potential causal factors and as a guide to postoperative rehabilitation.

\section{Protocols}

The goal of CPET protocols is to stress the organ systems involved in the exercise response in a controlled manner. For this reason the testing generally involves exercising large muscle groups, usually the lower extremity muscles. A key requirement is that exercise stimulus be quantifiable in terms of the external work and power performed. Simpler tests, such as step tests or timed distance walks (i.e., 6 or 12 min walk) can provide measures of exercise tolerance, but are not as useful in diagnosis as incremental tests [1, 2, 87]. The purpose of this section is to describe the characteristics of recommended clinical exercise protocols, both incremental and constant work rate tests.

\section{Standard clinical exercise protocol: incremental test}

The appropriateness of the integrated systemic responses to the tolerable range of work rates are best studied utilizing incremental exercise testing [88] (fig. 2). This provides a smooth gradational stress to the subjects so that the entire range of exercise intensities can be spanned in a short period of time. Technological advances (see section on equipment) have made it possible for sufficient density of data to be acquired in a test lasting less than $20 \mathrm{~min}$ (fig. 3), including: 1) measurements at rest; 2) 3 min of unloaded exercise; 3) incremental exercise (approximately $10 \mathrm{~min}$ ); and 4) 2 min recovery, at least. The recommended incremental exercise testing protocol, usually cycle or treadmill ergometry, is described in detail in the section on procedures. Electronically braked cycle ergometry with constant pedalling frequency, of 60 revolutions per minute (rpm) for example, is recommended (as discussed in the section on equipment). Equivalent results are obtained when work rate is either increased continuously (ramp 


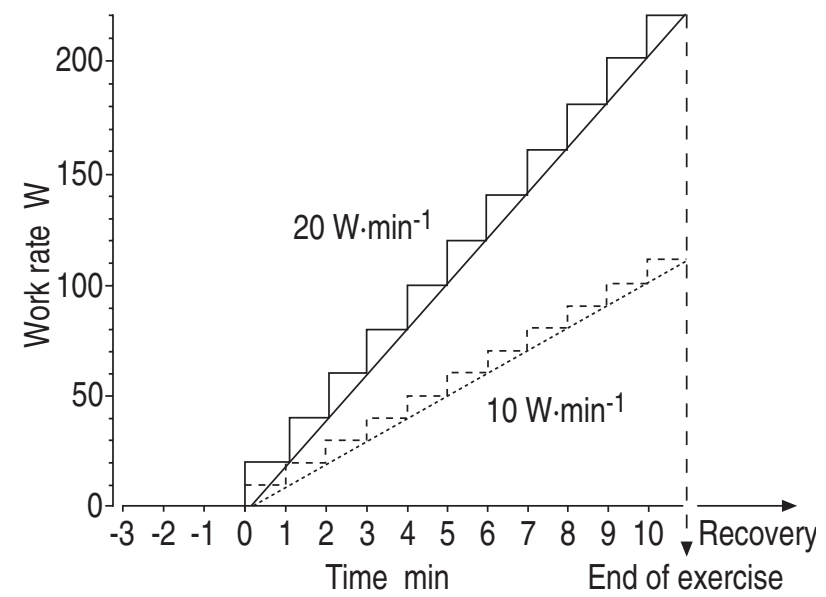

Fig 2. - Graphical representation of standard incremental exercise protocols. Equivalent results are obtained when work rate is either increased continuously (ramp test) or by a uniform amount each minute ( 1 min incremental test) until the patient is limited by symptoms (he/she can not cycle $>40 \mathrm{rpm}$ ) or is not able to continue safely. The increment rates of $10 \mathrm{~W} \cdot \mathrm{min}^{-1}(\ldots-)$ to $20 \mathrm{~W} \cdot \mathrm{min}^{-1}(-)$ is set according to the characteristics of the patient in order to obtain approximately $10 \mathrm{~min}$ duration of the incremental part of the protocol.

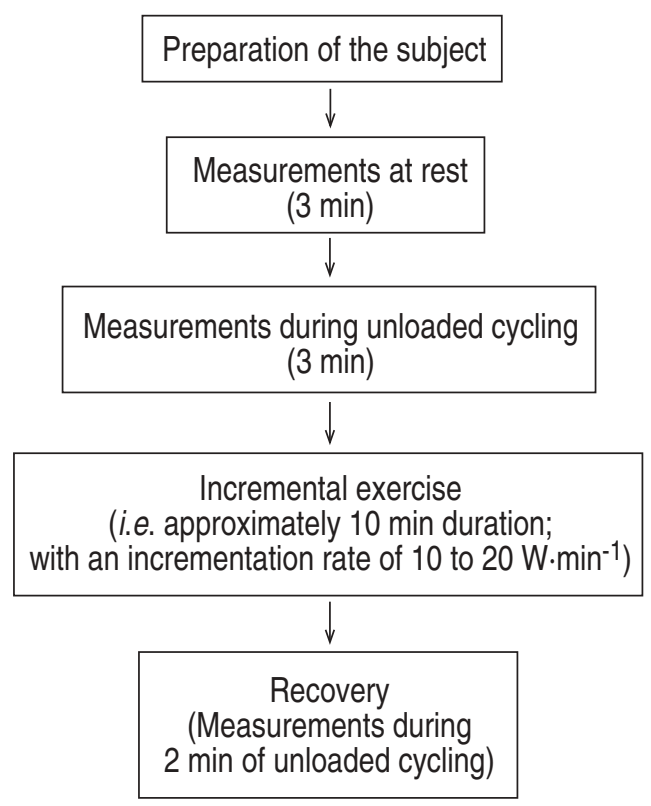

Fig. 3. - Recommended incremental exercise protocol using an electronically braked cycle ergometer.

test) or by a uniform amount each minute (1 min incremental test) until the patient is limited by symptoms (he/she cannot cycle $>40 \mathrm{rpm}$ ) or is not able to continue safely. The increment size should be set according to the characteristics of the patient in order to obtain approximately 10 min duration of the incremental part of the protocol. This may represent incrementation rates of $10-20 \mathrm{~W} \cdot \mathrm{min}^{-1}$ in a healthy sedentary subject or less in a patient $[1,2,87]$. Modifications in the design of the protocol and/or the measurements to be carried out during the test can be considered depending on: 1) the characteristics of the subject (physically fit or severely limited); and 2) the clinical problem prompting the indication of CPET (e.g., exercise-induced asthma, as described in detail by STERK et al. [89]).
If the ergometer is a motor-driven treadmill, then Balke's protocol $[90,91]$ is considered the most appropriate for its simplicity. The speed of the treadmill is kept constant $\left(5-6 \mathrm{~km} \cdot \mathrm{h}^{-1}\right)$ during the protocol while the slope is progressively increased $\left(1-2 \% \cdot \mathrm{min}^{-1}\right)$.

Standard noninvasive CPET carried out breathing room air $\left(F \mathrm{I}_{2} \mathrm{O}_{2}=0.21\right)$ involves acquisition of expired oxygen and carbon dioxide concentrations $\left(\mathrm{FE}, \mathrm{O}_{2}\right.$ and $F \mathrm{E}, \mathrm{CO}_{2}$, respectively), WR, respired airflow, $f \mathrm{C}$ and systemic arterial pressure as primary variables. ECG and pulse oximetry should be continuously monitored during the test. It is useful to establish a sense of the patient's exercise-related perceptions during the exercise test and at the point when the subject discontinues exercise. This includes exertion, dyspnoea, chest pain and skeletal muscle effort. Quantifying these perceptions should be done using standardized rating procedures (Borg scale, visual analogue scale (VAS), etc.) [92].

Interpretation of CPET results in patients with lung disease, however, often requires evaluation of pulmonary gas exchange [91]. In these cases, arterial cannulation (preferably radial or brachial) is needed to obtain partial pressures of respiratory gases in arterial blood $\left(\mathrm{Pa}, \mathrm{O}_{2}\right.$ and $\left.\mathrm{Pa}, \mathrm{CO}_{2}\right)$ and to determine DA-a, $\mathrm{O}_{2}$ [91, 93]. This also provides information on acid-base status $(\mathrm{pH}$, $P \mathrm{a}, \mathrm{CO}_{2}$ and base excess) and allows continuous monitoring of systemic arterial blood pressure during the test. However, while "arterialized venous blood" (e.g. from the dorsum of the heated hand) gives good values for $\mathrm{PCO}_{2}$ and $\mathrm{pH}$ it is not appropriate for $\mathrm{PO}_{2}$. Furthermore, estimation of arterial respiratory blood gases through expired oxygen and carbon dioxide profiles or "transcutaneous" electrodes and pulse oximetry should not be used as indices of $\mathrm{Pa}, \mathrm{O}_{2}$ and $\mathrm{Pa}, \mathrm{CO}_{2}$ during exercise [94-96]. It is important to recognize that arterial blood sampling immediately after exercise does not provide an adequate assessment of blood gas values at peak exercise. However, while pulse oximetry does not indicate arterial $\mathrm{Pa}, \mathrm{O}_{2}$, it does provide valuable information on oxyhaemoglobin saturation during exercise.

\section{Other tests}

Constant work rate exercise testing. Constant work rate exercise can result in steady-state responses when work rate is of moderate intensity (fig. 4). In contrast,

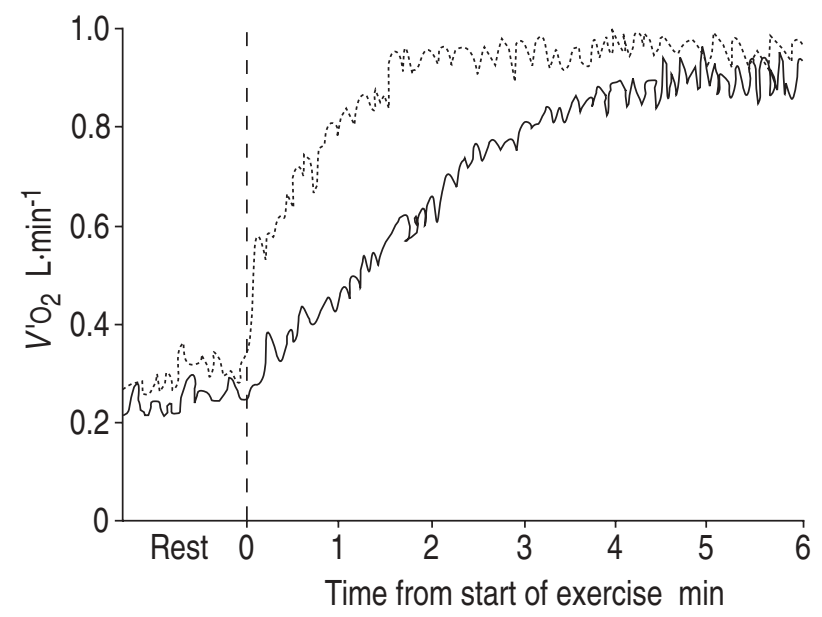

Fig. 4. - Time course of oxygen uptake during constant work rate exercise in one healthy subject (- - ) and one COPD patient (- 
a constant work rate of high intensity for the individual typically results in continually changing values in most variables of interest. Consequently, attainment of, or failure to attain, a steady-state $V^{\prime} \mathrm{O}_{2}$ during a constant-load test can be used to determine if a particular task is sustainable by the individual.

However, even for moderate exercise, steady-state cardiorespiratory responses do not occur instantaneously when exercise begins; rather, they change over a period of several minutes, but at rates which are different for each variable $\left(V^{\prime} \mathrm{O}_{2}, V^{\prime} \mathrm{CO}_{2}, f \mathrm{C}\right.$ and $\left.V^{\prime} \mathrm{E}\right)$. It has been demonstrated that both cardiopulmonary disease [13] and level of fitness [97] modify the time course of the nonsteady-state responses to exercise. Hence, the period of dynamic adjustment to a constant work rate test provides information regarding the dynamic behaviour of lung function, haemodynamics and tissue oxygen utilization. However, there is, to date, virtually no information on the confidence limits, reproducibility and predictive value of the derived parameters in patient populations. Consequently, the utility of quantifying dynamic responses to constant work rate exercise in clinical exercise testing remains to be established.

Some factors, however, such as the work efficiency or the substrate mixture undergoing oxygenation respiratory quotient $(\mathrm{RQ})$, require steady-state determination. It is necessary, in this case, to ensure that variables are measured only after the dynamic periods of adjustments are completed.

The $f \mathrm{C}$ during constant work rate testing is commonly used as a guideline in exercise prescription (rehabilitation programmes) based on the potential for self-monitoring and self-adjusting the exercise dose during training. Identification of a training effect following an exercise programme may also be based on the finding of a reduction in $f C$ for a given constant work rate.

Determining the need for oxygen supplementation during exercise or titrating oxygen prescription is most reasonably performed during submaximal constant work rate exercise, simulating levels of activity needed to perform daily activities [91]. Furthermore, because patients with COPD may be limited during exercise by impaired lung mechanics [2, 19, 26, 27], assessment of steady-state ventilation, and its pattern, might be of interest in evaluating the capacity for sustaining a given task.

It should be recognized that exercise recovery also follows a dynamic time course. Re-establishment of resting steady-state conditions following moderate exercise requires a similar time as for reaching the exercise steady-state, whereas recovery is delayed for considerably longer periods following high-intensity exercise. Even in young healthy subjects, resting levels of arterial blood lactate concentration, for example, are not achieved until approximately $1 \mathrm{~h}$ after maximum exercise.

Assessment of exercise-induced bronchoconstriction. Patients with reactive airways disease may experience bronchoconstriction following (or during) exercise. Exercise-induced bronchoconstriction (EIB) is observed in $70-80 \%$ of the population with clinically recognized asthma [89, 98, 99]. However, up to $40 \%$ of children with documented EIB have no symptoms of asthma
[100]. An exercise challenge is, therefore, often worthwhile for patients complaining predominantly of symptoms associated with exercise.

Respiratory water loss was identified as the stimulus by which exercise induces bronchoconstriction in the late 1970s and it was proposed that evaporative water loss induced cooling of the conducting airways causing them to narrow [101]. This later led to the proposal that EIB occurred as a result of vascular engorgement during rapid rewarming of the airways at the end of exercise [102]. Other studies, however, have demonstrated that the dehydrating effects of water loss are more important than the cooling effects. This led to the suggestion that an increase in airway osmolarity and release of mediators was the mechanism whereby the airways narrowed in response to exercise [103, 104]. At present, there is no direct experimental evidence to support either of these hypotheses.

However, these proposals led to the conclusion that tests for EIB should aim to achieve a high level of ventilation ( $V^{\prime} \mathrm{E}$ 15-22 times the predicted FEV1) while breathing air containing less than $10 \mathrm{mg} \cdot \mathrm{L}^{-1}$ of water (20-25 $5^{\circ} \mathrm{C}$ and $<50 \%$ relative humidity). Breathing compressed air via a valve will ensure low humidity of the inspired air. Recording ventilatory and gas exchange responses during the exercise period allows the level of $V^{\prime}$ E and metabolic stress to be documented.

The required $V^{\prime}$ E can be achieved either by running on a treadmill or by cycling [105]. Exercise performed with a cycle ergometer, however, is generally preferred because: 1) the breathing valve is more easily supported; 2) measurement of ventilation is easier; and 3) the work rate is largely independent of body weight. The target work rate can be estimated by equations relating $V^{\prime} \mathrm{E}$ to $V^{\prime} \mathrm{O}_{2}$ and $V^{\prime} \mathrm{O}_{2}$ to work rate. For treadmill exercise, body weight becomes a more important factor for consideration [106, 107] (table 2). The protocol recommended consists of a rapid increase in the work rate to the target value within 3-4 min and sustaining the target $V^{\prime} \mathrm{E}$ for at least $4 \mathrm{~min}$. For the first, second and third minutes of cycling the work rate may be conveniently set to 60,75 and $90 \%$ of the target value. At the beginning of the fourth minute the target work rate is set and $V^{\prime} E$ carefully monitored $[89,98]$.

In the patient with EIB, values for FEV1 generally fall to a minimum within the first 10 min after cessation of exercise, with substantial recovery by $30 \mathrm{~min}$ postexercise. A useful protocol to assess the response to exercise is to perform forced spirometry before (following recommended standardization) and then FEV1 manoeuvres at $1,3,5,7,10,15$ and 20 min after exercise. The response is assessed as the percentage fall in FEV1 postexercise. The lowest FEV1 recorded after exercise is subtracted from the pre-exercise value and the difference is expressed as a percentage of the pre-exercise FEV1. A greater than $10 \%$ fall in FEV1 after exercise is regarded as abnormal and $>15 \%$ as diagnostic of EIB. For a drug to be "protective", the fall in FEV1 after exercise should be less than $50 \%$ of that documented on placebo. The coefficient of variation of the exercise response (percentage fall in FEV1 after exercise) is $20 \%$ or less when two tests are performed under the same conditions within one month.

If the test is negative, it is important to check that: 
Table 2. - Conditions for evaluating the presence and severity of exercise-induced bronchoconstriction (EIB)

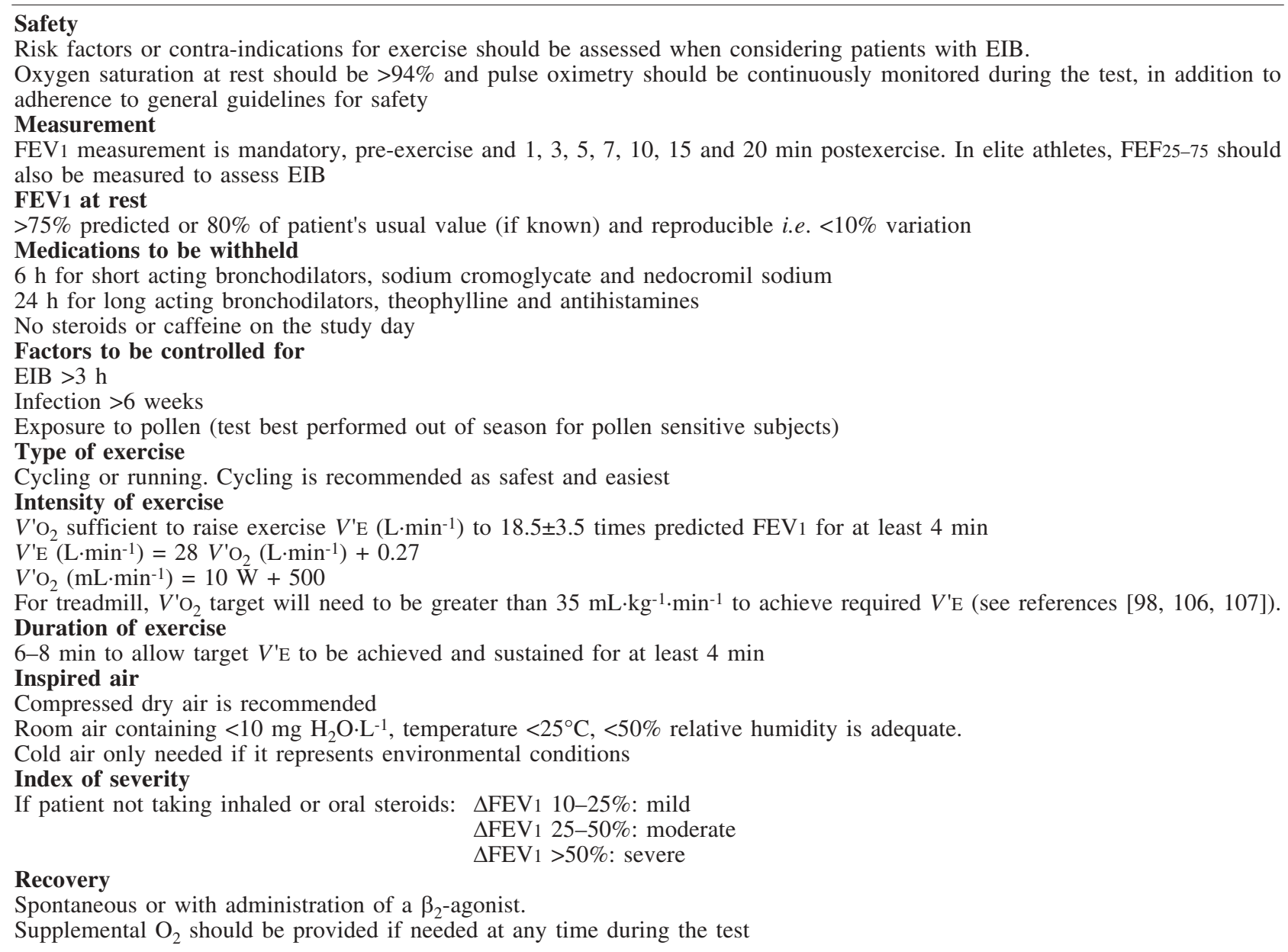

FEV1: forced expiratory volume in one second; $\mathrm{FEF}_{25-75}$ : forced midexpiratory flow; $V^{\prime} \mathrm{O}_{2}$ : oxygen consumption; $V^{\prime} \mathrm{E}$ : minute ventilation; $\Delta$ : change in.

1) the pre-exercise lung function was not unusually low; 2 ) the target $V^{\prime} E$ was achieved and sustained for at least $4 \mathrm{~min}$; and 3) the water content of the inspired air was $<10 \mathrm{mg} \mathrm{H} \mathrm{H}_{2} \mathrm{O} \cdot \mathrm{L}^{-1}$. While cooling the inspired air can increase the severity of the airway response in some subjects, this same effect appears to be achieved by prolonging the duration or increasing the intensity of exercise under temperate inspired air conditions [108]. It should be noted, however, that exercise itself is not required to achieve the target ventilation and voluntary eucapnic hyperventilation of air containing approximately $5 \%$ carbon dioxide can be performed [109, 110].

The protocol recommended is appropriate for both general laboratory assessment of EIB and evaluation of the protective effect of pharmacological agents [111, 112]. To maximize the likelihood of a positive response, bronchodilator agents, sodium cromoglycate and nedocromil sodium should be withheld prior to exercise for a period commensurate with their duration of action $[112,113]$. Furthermore, the subjects should avoid vigorous exercise for at least $2 \mathrm{~h}$ prior to testing. During the test, in addition to routine safety precautions required for all exercise testing, it is important to have rapidly acting inhaled bronchodilators (e.g. salbutamol) available to reverse a severe episode of bronchospasm.

\section{Equipment and quality-control programme}

\section{Choice of ergometers}

Two modes of exercise are commonly employed in cardiopulmonary exercise tests: treadmill and cycle ergometers. The motor driven treadmill is used to impose a progressively increasing exercise stress through a combination of speed and grade (slope) increases. Several incremental protocols are popular [90, 114-118]. During treadmill exercise testing a somewhat larger muscle mass can be brought to bear than in cycle ergometry, leading to a greater stress of the organ systems mediating the exercise response. On average, maximal oxygen uptake is reported to be $5-10 \%$ higher on a treadmill than on a cycle ergometer [119-122]. This may be important to detect abnormalities (i.e. cardiac ischaemia) that occur only with the highest metabolic demand. The main disadvantage of using a treadmill is the difficulty of quantifying the work rate accurately. The relationship between speed and grade of the treadmill and the metabolic cost of performing work is difficult to predict: the "walking skill" and pacing strategy are important determinants. Holding on to the treadmill handrails can substantially 
modify the metabolic cost of treadmill walking, but by an unpredictable amount. These uncertainties make the profile of $V^{\prime} \mathrm{O}_{2}$ increase difficult to interpret. The relationship of other variables to $V^{\prime} \mathrm{O}_{2}$, however, remains appropriate.

For laboratory exercise testing, there are several advantages in using a cycle ergometer. It is generally cheaper and requires less space. It is less prone to introduce movement or noise artefacts into measurements (i.e. blood pressure auscultation is generally easier). The most important advantage, however, is that the rate at which external work is performed is much more easily quantified. Although the metabolic cost of moving the legs at ostensibly zero load is a confounding factor [2, 123-125], this represents a constant offset as long as the pedalling cadence is kept constant. The predictability of the relationship between the imposed cycle ergometer work rate and the metabolic energy expenditure is important for disease evaluation.

The electronically-braked cycle ergometer [126] is generally used in CPET. Friction-braked cycle ergometers [127] generally provide a work rate that varies with the cycling frequency. In contrast, the electronically braked cycle ergometer allows direct quantification of the work rate performed, which is usually independent of cycling frequency. It can also be readily computer controlled; this allows the work rate to be incremented continuously (ramp exercise) [88, 128-132]. Recently, cycle ergometers have become available that allow "true unloaded" pedalling (i.e. internal resistance of the ergometer is overcome by means of a motor "assist"). A lower starting work rate can therefore be selected; this is important for the most debilitated patients.

\section{Gas exchange measurements}

A central focus of comprehensive CPET is the interpretation of gas exchange responses. Consequently the methods used to measure $V^{\prime} \mathrm{O}_{2}$ and carbon dioxide output $V^{\prime} \mathrm{CO}_{2}$ are of important. However, these measurements are not trivially easy to make, and a clear understanding of the methods involved and the required quality control procedures are necessary prerequisites.

The technologically simplest technique of measuring gas exchange involves directing the expired air into a collection bag by use of a suitable breathing valve. A timed collection is made; the mixed concentrations of carbon dioxide and oxygen in the bag and the volume of the bag are subsequently measured. This allows calculation of $V^{\prime} \mathrm{O}_{2}$ and $V^{\prime} \mathrm{CO}_{2}$ [91, 133, 134]. By convention, $V^{\prime} \mathrm{O}_{2}$ and $V^{\prime} \mathrm{CO}_{2}$ are expressed under standard temperature and pressure, dry (STPD) conditions while ventilation is expressed at body temperature and pressure, saturated with water vapour (BTPS) (for complete definitions of STPD and BTPS, see Appendix). Use of highly accurate gas analysers is, naturally, essential. Though careful attention to technique is crucial, this method is capable of very precise measurements even at high metabolic rates. However, it is less suitable for rapid incremental exercise protocols as it is cumbersome and the resulting data density is relatively low. Bag collection systems are now mainly used as a validation technique for more complex gas exchange systems.
Systems featuring mixing chambers allow continuous measurement of $V^{\prime} \mathrm{O}_{2}$ and $V^{\prime} \mathrm{CO}_{2}[2,135,136]$. The subject respires through a breathing valve and expired air is directed through a baffled chamber. The concentrations of carbon dioxide and oxygen are measured continuously at the distal end of the mixing chamber. Expired volume is measured, usually breath-by-breath. $V^{\prime} \mathrm{O}_{2}$ and $V^{\prime} \mathrm{CO}_{2}$ can then be calculated from the gas concentration signals. In the steady-state of exercise, mixing chamber systems are capable of accurate metabolic measurements. However, since the washout of the mixing chamber requires a finite time (which depends on the airflow), the volume and gas concentration signals will be misaligned in the unsteady-state, leading to inaccurate calculations unless appropriate corrections are made. For incremental protocols commonly used in clinical CPET, ventilation and mixed expired gas concentrations do not change rapidly and the accuracy of a well designed mixing chamber system may be quite acceptable (though care must be taken to recognize that slurring of the response profile is a necessary consequence not only when variables change rapidly but also when they change direction (i.e., expired partial pressure of oxygen $\left(P \mathrm{E}, \mathrm{O}_{2}\right)$ at the lactate threshold).

With the ready availability of on-line digital computer analysis of physiological transducer signals, it has become practical to compute $V^{\prime} \mathrm{O}_{2}$ and $V^{\prime} \mathrm{CO}_{2}$ breath-bybreath [137-141]. Utilizing algorithms first reported in 1973 [137], a signal proportional to expired airflow and signals proportional to fractional concentrations of carbon dioxide and oxygen measured near the mouth are sampled at high frequency (typically $50-100 \mathrm{~Hz}$ ). As shown schematically in figure 5 , the breath is broken down into a number of parts and the $V^{\prime} \mathrm{CO}_{2}$ and $V^{\prime} \mathrm{O}_{2}$ computed for each interval. The measurements for each interval are summed over the expiration to compute the total volume of carbon dioxide exhaled and oxygen taken up in the breath.

$$
\begin{gathered}
V^{\prime} \mathrm{CO}_{2}=\Sigma F \mathrm{E}, \mathrm{CO}_{2} \cdot V^{\prime} \mathrm{E} \cdot \Delta \mathrm{t} \\
V^{\prime} \mathrm{O}_{2}=\Sigma\left(F \mathrm{I}, \mathrm{O}_{2}-F \mathrm{E}, \mathrm{O}_{2}\right) \cdot V^{\prime} \mathrm{E} \cdot \Delta \mathrm{t}
\end{gathered}
$$

where $V^{\prime} \mathrm{E}$ is the instantaneous expired airflow, $\Delta \mathrm{t}$ is the sampling interval and $\mathrm{FI}, \mathrm{O}_{2}, F \mathrm{E}, \mathrm{O}_{2}$ and $\mathrm{FE}, \mathrm{CO}_{2}$ are the fractional concentrations of inspired oxygen, expired

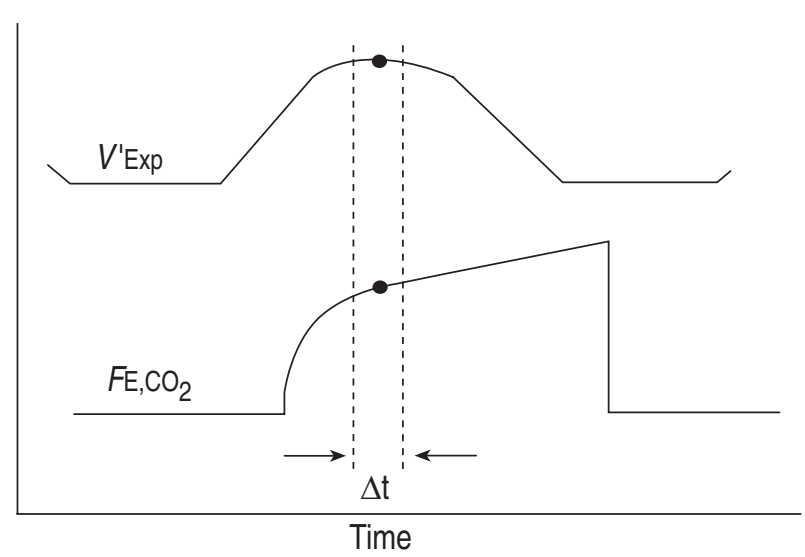

Fig 5. - Diagram illustrating calculation of breath-by-breath carbon dioxide output $\left(V^{\prime} \mathrm{CO}_{2}\right)$, which is derived from $V^{\prime} \mathrm{CO}_{2}=\sum F \mathrm{E}, \mathrm{CO}_{2}$. $V^{\prime} \mathrm{E} \cdot \Delta \mathrm{t} V^{\prime}$ Exp: expired airflow; $F \mathrm{E}, \mathrm{CO}_{2}$ : expired $\mathrm{CO}_{2}$ fraction; $\Delta \mathrm{t}$ : sampling interval. 
oxygen and expired carbon dioxide, respectively. These calculations must accommodate water vapour, barometric pressure and ambient temperature variations in order to obtain STPD values in addition to the "nitrogen correction factor" when only expired volumes are measured (see Appendix). As importantly, compensation is necessary for the delay between the time at which gas is sampled at the mouth and the time at which the gas concentration is measured within the gas analysers (usually in the order of $0.25 \mathrm{~s}$ ). Breath-by-breath analysis, therefore, requires precise knowledge of (and computerized compensation for) the transport delays of both gas analysers [140, 142-144]. Thus, the increased temporal resolution of breath-by-breath analysis comes at a cost of more exacting quality control requirements.

Measurement of oxygen uptake when $\mathrm{FI}_{\mathrm{I}} \mathrm{O}_{2}$ is high presents particular problems. First, the source of inspired gas must have a constant oxygen fraction (i.e., from large gas bags). Further, when $F \mathrm{I}_{1} \mathrm{O}_{2}$ approaches 1 , nitrogen balance corrections become more and more subject to error [142]. Depending on the methodology employed and the accuracy required, $F \mathrm{I}_{1} \mathrm{O}_{2}$ of 0.8 or more should be regarded as beyond the practical upper limits of accurate measurement of $V^{\prime} \mathrm{O}_{2}$.

\section{The lactate threshold}

Although there is no generally agreed upon procedure for normalizing work intensity, most would concede that the range of work rates within which there is not a sustained metabolic (chiefly lactic) acidemia (i.e., moderate exercise) may be sustained for long periods, but heavier exercise may not [2]. The threshold $V^{\prime} \mathrm{O}_{2}$ for arterial lactate concentration increase (the lactate threshold (LT)) may, therefore, be considered to partition exercise into moderate and heavy intensities, with important implications for the ability to sustain a particular work rate. However, it should be emphasized that physiological mechanisms underlying the increases in muscle and blood lactate concentration that occur above LT remain a highly contentious issue; some investigators even question whether the arterial lactate concentration profile actually provides evidence of threshold behaviour [97, 145]. The lactate threshold may, therefore, be considered to: 1) partition moderate from heavy-intensity exercise; 2) trigger a series of physiological responses that stress ventilation, pulmonary gas exchange, and acid-base regulation; and 3) have important implications for the ability to sustain muscular exercise, both in normal individuals and in patients with impaired systemic function.

Determination of LT. The lactate threshold is highly task-specific. It occurs at an appreciably lower $V^{\prime} \mathrm{O}_{2}$ for arm exercise than for leg exercise, and is typically lower for cycle ergometry than treadmill exercise, reflecting the magnitude of muscle mass over which the work is distributed. A wide range of techniques have been advocated for estimation of LT, including both direct measurements and indirect estimation [91]. The description of the direct measurements of LT [146, 147] (arterial lactate concentration-pyruvate concentration ratio; logarithmic arterial lactate concentration and bicarbonate concentration functions) is beyond the scope of the present document.

One can forego the necessity for serial blood sampling and even, in many cases, enhance the discriminability of LT by utilizing a particular cluster of ventilatory and pulmonary gas exchange responses, which provide noninvasive estimation of LT (V-slope method or time course of ventilatory equivalents for oxygen and carbon dioxide; see Appendix). However, the indiscriminate use of noninvasive estimators of LT should be discouraged. Without the supporting evidence that the more rapid rate of change of $V^{\prime} \mathrm{CO}_{2}$ or $V^{\prime} \mathrm{E}$ is a consequence of the developing metabolic acidemia, the change could also reflect any of a range of hyperventilatory causes of particular concern in patients with lung disease.

Furthermore, the validly of the noninvasive estimation of LT under "complicating" conditions such as chronic hyperventilation, progressive exercise-induced hypoxaemia, and impaired peripheral chemosensitivity with an associated high airway resistance remains to be established.

\section{Exercise testing measurement systems}

High intensity data analysis and display of a range of relevant variables (e.g. [2]) is most likely to provide patterns of response that optimize discrimination (especially of subtle differences). Tests using less complex, and certainly less expansive equipment and procedures can also provide useful information, however, especially if the issue in question is well focused (see [116] for discussion). Figure 6 presents a schematic representation of the analytical devices that constitute a recommended measurement system for CPET. The following components are usually present: 1) digital computer; 2) flow or volume measurement system; 3) gas analysers; 4) electrocardiograph (ECG); and 5) pulse oximetry. Measurement of systemic arterial blood pressure and analysis of respiratory blood gases $[93,94]$ and acid-base status [2,148] should also be considered part of the basic CPET equipment.

Digital computer. The analogue-to-digital converter needs to sample a number of signals at $50-100 \mathrm{~Hz}$ and the computer should be capable of computations of variables within each sampling interval. A key function is

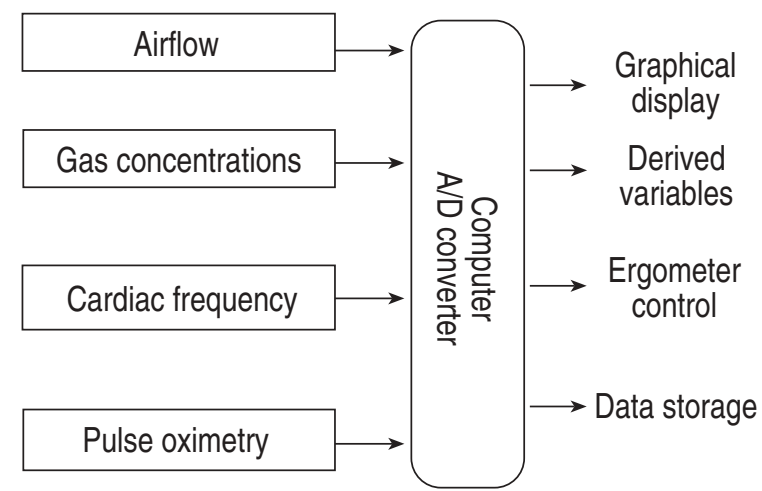

Fig. 6. - Schematic representation of the equipment used for cardiopulmonary exercise testing (CPET). A/D: analogue/digital. 
breath detection, often signalled by the detection of the onset of expiratory airflow. The breath-by-breath values of a number of variables should be available for on-line graphical or tabular display and should be stored for later analysis.

Flow or volume measurement of respired air. A number of transducers have been used for measurement of respired flow or volume during exercise. In part, the expansion of the choice of transducers has resulted from the use of computerized data analysis. A nonlinear relationship between the actual flow or volume and transducer output can be accommodated by digital computer processing, as long as the nonlinearity has been well characterized and is static (i.e., the relationship does not vary with time). Furthermore, the choice of a flow versus a volume transducer is no longer crucial since numerical integration or differentiation can be employed to calculate one quantity from the knowledge of the other. A key consideration is whether the transducer can be positioned near the mouth. Such transducers are capable of sensing bi-directional flow or volume. They also eliminate the need for a nonrebreathing valve which means that the apparatus dead space can be lower. Both the ERS and the American Thoracic Society (ATS) have established standards for flow and volume measurement in the context of spirometry [149, 150] (table 3). The transducers used in CPET should also meet these standards. Transducers commonly employed for measuring flow or volume in CPET are listed below.

Pneumotachograph. This flow transducer measures the pressure drop across a low resistance screen $[2,119$, $151,152]$. Since laminar flow and constant temperature are required and sputum impaction on the transducer screen can degrade performance, pneumotachographs have generally been positioned well downstream from the mouth. The latter two concerns, however, can be avoided by measuring inspired rather than expired airflow.

Pitot tube flowmeter. This device measures the difference between pressure at orifices facing the flow stream and orifices perpendicular to the flow stream. Turbulent (rather than laminar) airflow is involved and the pressure difference is proportional to the square of the flow rate $[2,153]$.

Mass flow meter. This device is related to the older hot wire anemometer in which a wire is placed in the airstream. The current required to heat the wire to a certain temperature increases as airflow increases [154, 155]. In one configuration presently used, two wires heated to different temperatures are utilized; flow detec- tion depends on the fact that the hotter wire loses heat more rapidly than the cooler wire. Compensation is made electronically for changes in gas temperature. The signal generated is (nonlinearly) proportional to the number of molecules passing the sensor rather than the volume of gas the molecules occupy.

Turbine volume transducer. A lightweight impeller is placed in the flow stream and the number of interruptions of a light beam by the impeller are counted by a computerized system [156]. Although small, the mass of the impeller causes a small "start up" and "overrun" at the start and end of the breath, respectively. The dynamic nonlinearities can lead to errors in the timing of the breath [157].

Gas analysers. Different strategies have been employed to measure the gas concentrations necessary for breath-by-breath analysis. One is to use an analyser capable of measuring all the relevant gases (carbon dioxide, oxygen and, for some purposes, nitrogen). Mass spectrometry has most often been used for this purpose. The other approach utilizes separate analysers for each gas species. Key requirements are stability and rapidity of response. The dynamics of analyser response has two separate components: transport delay (the time required for the gas to traverse the distance from the sampling site to the analyser) and analyser response (the kinetics of response to a change in gas composition introduced into the analyser). Transport delay (generally in the order of $0.2-0.5 \mathrm{~s}$, depending on the length of the gas sampling tube and the gas sampling rate) can be readily compensated for. The analyser response, often taking the form of an exponential or sigmoid response to a stepwise change in gas composition [138, 139] must be kept as short as possible and included in the total "delay". An additional concern is the sensitivity of the analyser to the partial pressure of water vapour in the sampled gas. Since water vapour concentration in the sampled gas can be difficult to predict (principally because the gas temperature at the sampling point is difficult to predict), this can introduce substantial errors in metabolic rate calculations [134].

The mass spectrometer ionizes gas molecules in a high vacuum environment and then separates them (by one of several schemes) on the basis of mass to charge ratio. This enables the time courses of a number of gas species to be measured. These analysers are linear, often highly stable and have rapid response characteristics (analyser time constants of roughly $25-50 \mathrm{~ms}$ ). They are usually configured to "ignore" water vapour, yielding dry gas fractions. However, the high cost of mass spectrometers has inhibited their use in most commercial CPET systems.

Discrete carbon dioxide and oxygen analysers have

Table 3. - Minimum requirements of the equipment

\begin{tabular}{llcccc}
\hline Equipment & Range & Accuracy* & Reproducibility & Frequency response \\
\hline $\mathrm{O}_{2}$ analyzer & $0-100 \%$ & $1 \%$ & $1 \%$ & $<130 \mathrm{~ms}$ & At least, two point calibration \\
$\mathrm{CO}_{2}$ analyzer & $0-10 \%$ & $1 \%$ & $1 \%$ & $<130 \mathrm{~ms}$ At least, two point calibration \\
Flow meter & $0-14 \mathrm{~L} \cdot \mathrm{min}^{-1}$ & $3 \%$ & $3 \%$ & $<40 \mathrm{~ms}$ & $3 \mathrm{~L}$ syringe \\
Cycle ergometer & $0-600 \mathrm{~W}$ & $2 \%$ or $3 \mathrm{~W}$ above $25 \mathrm{~W}$ & - & - & Dynamic torquemeter
\end{tabular}

*: linearity within the indicated percentage of full scale for each apparatus. 
been designed specifically for the demands of cardiopulmonary exercise testing. Carbon dioxide analysers, based on absorption of infra-red light by carbon dioxide [158], are common. The oxygen analysers that are employed are different, based on two principles. In paramagnetic analysers, the effect of oxygen molecules on a magnetic field is utilized. In the electrochemical ("fuel cell") analyser, high temperature reactions between oxygen and substrate are measured. However, these discrete oxygen and carbon dioxide analysers have potential disadvantages. While the analyser output is usually not a linear function of gas concentration, computerized correction for these nonlinearities can be made. Also, analyser measurements are influenced by water vapour concentration in the sampled gas. This problem has been circumvented by using sampling tubing composed of the polymer Nafion. This polymer contains sulphydryl residues that absorb water, allowing water molecules to be transported down its concentration gradient to the exterior of the tubing. The gas that reaches the analyser contains little water vapour.

$E C G$. Serial 12-lead ECG is optimal in subjects undergoing CPET for detection of disease. It is important to detect ST-segment and T-wave changes consistent with ischaemia and to define arrhythmias that occur with exercise [127, 159]. Three-lead ECG recordings for detection of cardiac frequency are suitable in young subjects in whom CPET is being used principally to define exercise tolerance. A key requirement is that electrodes should be properly placed using sweat resistant adhesive patches and the detection electronics be specially designed for movement artefact rejection. Computerized systems enabling continuous display contribute to test safety; averaging of ECG complexes can improve detection of ischaemic changes. Extensive description of electrode placement in 12-lead ECG and requirements of ECG recording in CPET can be found elsewhere [160].

Pulse oximetry. The current generation of pulse oximeters detect the variation in transmission of light of two different wavelengths that occur with arterial pulses in an extremity (usually the finger or ear lobe). Because oxygenated and reduced haemoglobin transmit certain wavelengths of light differently, this information can be used to estimate arterial oxygen saturation $\left(\mathrm{Sa}_{\mathrm{a}} \mathrm{O}_{2}\right)$ [94]. While useful and convenient for continuous monitoring $[161,162]$, several concerns need to be kept in mind in the context of exercise testing. Pulse oximeters have limited accuracy (95\% confidence limits of $\pm 4-5 \%$ as compared to directly measured $\mathrm{Sa}_{\mathrm{a}} \mathrm{O}_{2}$ ) [163]. Some authors have reported that pulse oximeters tend to overestimate true $\mathrm{Sa}_{\mathrm{a}} \mathrm{O}_{2}$ values [164-166], a particular problem when test results are being used to prescribe oxygen therapy. On the other hand, poor perfusion of the extremity (yielding decreased pulsatility), which may occur in cardiovascular disease, may yield falsely low readings [167]. Movement and stray light can yield artefacts and dark skin colour can interfere with signal detection [168, 169]. Furthermore, the inherent limitations of pulse oximetry must be appreciated [91]. These devices cannot detect the effects of increased carboxyhaemoglobin (or methaemoglobin); its calculations appro- ximate the oxygenated fraction of available haemoglobin. An additional disadvantage of pulse oximetry is that $\mathrm{Sa}, \mathrm{O}_{2}$ rather than $\mathrm{PO}_{2}$ is measured. $\mathrm{Pa}, \mathrm{O}_{2}$ (and the derived variable DA-a, $\mathrm{O}_{2}$ ) are more relevant in assessing the effects of lung disease on pulmonary gas exchange.

Systemic arterial blood pressure. Auscultation of blood pressure becomes more difficult during exercise because of the increase in ambient noise. Yet detection of exercise induced hypertension (or, less commonly, hypotension) is an important goal in many circumstances [127]. Automated blood pressure measurement systems have been developed specifically for use during exercise. Many operate with the oscillometric method, in which, as the cuff is automatically deflated in stages, pressure oscillations induced within the cuff by pulsations in the arm are detected [170]. Despite algorithms designed to decrease the effects of artefacts, blood pressure measurements may be inaccurate when, for example, the arm moves during the measurement cycle. Periodic checks against manual determinations are important.

For studies in which a radial or brachial artery catheter is inserted to facilitate blood sampling, it may be useful to measure blood pressure directly. It should be appreciated that there are (modest) systematic differences between auscultated and intra-arterial blood pressure measurements [121, 171]. Miniature transducers that can be attached to the arm while the subjects exercises are available. Meticulous attention to technique (i.e., exclusion of air bubbles) is necessary, as elsewhere with CPET, to ensure a good frequency response. Sterility concerns have led some laboratories to use single-use disposable blood pressure transducers. Proficiency guidelines for the analysis of arterial blood gases should be followed.

\section{Quality control programme}

For CPET systems purchased as a unit, the manufacturer should be required to bear responsibility for demonstrating that the system is capable of accurate measurement. This might include description of bag collection comparisons over a range of metabolic rates and with a variety of breathing patterns. Algorithms used for breath detection and for calculation of variables should be provided to the user. However, it must be stressed that the user bears the responsibility for ensuring that measurements are accurate and reproducible on a day-to-day basis. CPET, especially when it features breath-by-breath gas exchange analysis, requires meticulous attention to calibration procedures to ensure accurate and reproducible measurements (table 3 ). A good practice is to maintain a calibration log book so that long term trends can be monitored and sudden changes recognized and addressed.

Daily calibration begins with the determination of ambient barometric pressure, temperature and relative humidity. Several other calibration manoeuvres are performed daily (or more often if many tests are done) to check the operation of key transducers. Most commercial exercise systems facilitate these calibrations.

Verification of calibration of the airflow or volume transducer can be performed with a precision large volume syringe (e.g. $3 \mathrm{~L})$. A wide range of flow rates 
should be performed to simulate the range of flows that occur between rest and heavy exercise; syringe strokes varying from $1-10 \mathrm{~s}$ in duration cover most of this range. Agreement in calculated volumes to within $\pm 3 \%$ signifies adequate performance [149, 150].

Although the output of most carbon dioxide and oxygen gas analysers is a nonlinear function of gas concentration, electronic algorithms aim to create linear outputs over the desired operating range. For carbon dioxide, this is usually $0-8 \%$; for oxygen it is $13-21 \%$ (unless testing with hyperoxic gas mixtures is intended). At least daily, a minimum of two point calibrations of each analyser (with at least two precision gas mixtures) should be performed. On occasion, certified tanks with other relevant compositions should be used to verify linearity. This is conveniently done with: 1) one $3 \%$ carbon dioxide and $13 \%$ oxygen tank; 2 ) one $6 \%$ carbon dioxide and $17 \%$ oxygen tank; and 3 ) one $0 \%$ carbon dioxide and $21 \%$ oxygen tank. Few laboratories nowadays are capable of performing Scholander analysis [172]; mass spectrometry is a reasonable "gold standard" measurement modality. A good practice is to maintain a single precision gas cylinder for occasional use; such a tank can last a number of years and (if regularly rotated to minimize settling effects) provide a long-term validation of calibration accuracy.

A third routine calibration must be performed in systems featuring breath-by-breath gas exchange measurements. The transport delay between the gas sampling point and the gas analysers needs to be known with precision so that the respiratory airflow and gas concentration signals can be properly time aligned. A solenoid allows an abrupt switch between two gas sources with different oxygen and carbon dioxide compositions and the time delay can be measured between the solenoid activation and the detection of change in gas analyser output. To this delay is added one time constant if the analyser response is exponential or the half time if the response is sigmoidal.

It is useful to perform an overall check of the ventilation and gas exchange measurements on a regular basis. One approach is to use a gas exchange simulator [173] that features a reciprocating piston; injection of a precision gas mixture at a precisely metered rate yields simulation of a known $V^{\prime} \mathrm{E}, V^{\prime} \mathrm{CO}_{2}$ and $V^{\prime} \mathrm{O}_{2}$. Day-to-day variation of these calculations and the variation with changes in pump rate should be roughly in the range of $\pm 3 \%$. It should be noted that this gas exchange simulator does not simulate the moist exhalate at the expired temperature. Thus, assumptions about temperature and humidity corrections are not tested.

Other calibration procedures also need to be performed. Blood pressure transducers can be calibrated with a mercury manometer. Devices are available to calibrate electromagnetically braked cycle ergometers [174176]; calibration should be performed at least every 6 months or whenever the cycle ergometer is moved (jarring often disturbs the calibration) or when an unusual response profile raises concerns about the equipment. For treadmills, belt speed should be verified by timing revolutions of the belt with a subject on the treadmill; accuracy of the grade indication should also be validated [127]. There are two other quality control checks that are advisable. A healthy member of the laboratory staff (with a consistent diet) should perform a constant work rate test at regular (perhaps weekly) intervals. Steady state values for $V^{\prime} \mathrm{O}_{2}$ differing by more than perhaps $7 \%$ from previous values should engender a thorough system-wide reassessment.

Finally, timed expired gas collections made during the steady state of exercise can be used as a "gold standard" to validate ventilation and gas exchange measurements. Though laborious, when performed carefully, this method is generally accurate to within $2-3 \%$.

\section{Personnel and testing procedures}

\section{Personnel qualifications}

Cardiopulmonary exercise testing should be conducted only by adequately trained personnel with a basic knowledge of exercise physiology. Technicians familiar with normal and abnormal responses during exercise and trained in cardiopulmonary resuscitation (CPR) should be present throughout the test. CPET should be performed under the supervision of a physician who is appropriately trained to conduct exercise tests and in advanced CPR. The degree of subject supervision needed during the test can be determined by the clinical status of the subject being tested and the type of exercise protocol. While it is preferable for the physician to be present during the test, if it is not possible, he/she must be readily available to respond as needed. Additional roles for the physician are the evaluation of the patient immediately before the test and the interpretation of the results.

\section{Patient preparation}

At the time of scheduling, the subject should be instructed to adhere to his/her usual medical regimen; he/ she should not eat for at least $2 \mathrm{~h}$ before the test, avoid cigarette smoking and caffeine, and dress appropriately for the exercise test. A brief history (with detailed inquiries about the medications) and physical examination should be taken to rule out contra-indications to testing. Results of recent resting pulmonary function tests (as a minimum forced spirometry) should be available for patients in whom pulmonary disease is suspected.

On arrival at the CPET laboratory, a detailed explanation of the testing procedure and equipment should be given to the patient, outlining risks and potential complications as described below. The subject should be told how to perform the exercise test and the testing procedure should be demonstrated, if needed. The patient should be encouraged to ask questions to reduce any anxiety. The patient needs to become familiar with the equipment [91]. If the treadmill is used, time is provided for several practice trials of starting and stopping until the patient feels confident. If the cycle ergometer is used, the seat height is adjusted so that the subject's legs are almost completely extended when the pedals are at the lowest point and the cycling rhythm practiced. 
If arterial blood sampling is required, a catheter is inserted into a distal artery (preferably the radial or brachial artery) of the nondominant arm following proper procedures to ensure collateral circulation. Sterile technique must be utilized during the catheter placement [148, 177]. A continuous flush device must provide a slow infusion of a heparinized saline solution. The dead space of the catheter used for sampling should be less than 1 $\mathrm{mL}$. To avoid spurious dilution, $2 \mathrm{~mL}$ of blood is discarded before collecting each arterial blood sample.

Before the test, the ECG electrodes are carefully placed and secured after preparing the skin to ensure good recordings (if necessary, the area of the electrode placement should be shaved). A sphygmomanometer cuff is placed on the upper arm. The mouth piece and nose clip are then tried and the position adjusted until a comfortable position is adopted. The patient is informed that it is acceptable to swallow with the mouth piece in place and that he/she must signal any unexpected difficulty by the signal "thumbs down". The patient is advised to point to the site of discomfort if chest or leg pain is experienced.

During the test, the patient is encouraged to carry on with a regular pedalling rhythm. Use of a metronome to assist in maintaining rhythm is often helpful. Symptoms and degree of discomfort are checked periodically (see safety precautions below). Good communication with the patient throughout the whole procedure increases the subject's confidence and predisposes to good effort.

During recovery, the patient is told to continue to pedal, without external workload (or walk at a slow pace on the treadmill), for at least 2 min during recovery in order to prevent fainting and to accelerate lactate removal. At the point when the subject discontinues exercise, after removal of the mouthpiece, the physician should ask for symptoms (type and intensity) that prompted the patient to stop exercise. If blood gas analysis is performed, a last blood sample is taken after 2 min of recovery.

If the test does not provide adequate diagnostic information because of premature termination or inadequate co-operation of the patient, it should be repeated after a resting period of 30-45 min.

After removal of the radial (or brachial) artery catheter, adequate pressure must be applied for sufficient time to avoid bleeding. This can take up to $10-15 \mathrm{~min}$. A compression bandage should be applied to the site of the puncture. The patient is advised not to remove the bandage and not to use his arm for heavy exercise within $6 \mathrm{~h}$ after the test.

\section{Safety precautions}

Although CPET may be considered to be a safe procedure, risks and complications have been reported. A recent review [160] summarizing eight studies of estimates of sudden cardiac deaths during exercise testing revealed rates from none (four studies) to five per 100,000 tests. A French study [178] based on 458,000 exercise tests (1975-1985) reported one death per 76,000 exercise tests. However, the relative risk of an adverse event is strongly related to the underlying disease, particularly in subjects post-myocardial infarction or with malignant arrhythmias. Good clinical judgement should be paramount in defining indications and contra-indications for exercise testing [159]. Table 4 lists absolute and relative contra-indications to CPET. Cardiac (bradyarrhythmias, ventricular tachycardia, myocardial infarction, heart failure, hypotension and shock) and noncardiac (musculoskeletal trauma, severe fatigue, dizziness, fainting, body aches) complications of CPET have been reported. Consequently, during the test, the personnel should be alert to any abnormal event.

Indications to stop the test must be clearly established and known by all the personnel involved in testing. These indications include symptoms such as: 1) acute chest pain; 2) sudden pallor; 3) loss of co-ordination; 4) mental confusion; and 5) extreme dyspnoea. The signs include: 1) depression of ST segment greater than 0.1 $\mathrm{mV}$ (less specific in females); 2) T-wave inversion; 3) sustained ventricular tachycardia; and 4) fall in systolic pressure either below the resting value or about 20 $\mathrm{mmHg}$ below its highest value during exercise testing. Relative indications to stop the test are: 1) polymorphic and/or frequent premature ventricular beats; and 2) hypertension $(>250 \mathrm{mmHg}$ systolic; $>130 \mathrm{mmHg}$ diastolic). If the exercise test has been stopped for one of these reasons, the patient should be monitored in the CPET laboratory until symptoms or ECG modifications have completely cleared. Admission to hospital for longer observation or more often for complementary investigation will be necessary in very rare cases. If necessary, intensive care can be administered on site. Full CPR equipment should be available in the CPET laboratory.

Table 4. - Contra-indications for cardiopulmonary exercise testing

\begin{tabular}{ll}
\hline Absolute & Relative \\
\hline Acute myocardial infarction (3-5 days) & $\begin{array}{l}\text { Left main coronary stenosis or its equivalent } \\
\text { Unstable angina }\end{array}$ \\
$\begin{array}{l}\text { Uncontrolled arrhythmias causing symptoms or haemodynamic } \\
\text { compromise }\end{array}$ & Sectrolyte abnormalities \\
Active endocarditis & systolic, $>120 \mathrm{mmHg}$ diastolic) \\
Acute myocarditis or pericarditis & Significant pulmonary hypertension \\
Symptomatic severe aortic stenosis & Tachyarrhythmias or bradyarrhythmias \\
Uncontrolled heart failure & Hypertrophic cardiomyopathy \\
Acute pulmonary embolus or pulmonary infarction & Mental impairment leading to inability to cooperate \\
Acute noncardiac disorder that may affect exercise performance or & High-degree of atrioventricular block \\
be aggravated by exercise $(i . e$. infection, renal failure, thyrotoxicosis) & \\
Thrombosis of lower extremities &
\end{tabular}

"Relative" contra-indications can be over-ruled if the benefits outweigh the risks of exercise. 


\section{Interpretative strategies}

As mentioned above, the main general goal of CPET is to evaluate the degree of limitation of exercise tolerance and to identify its causal factors. Optimal clinical application of CPET should be attained by properly accomplishing each of the steps indicated in figure 1: 1) identify the clinical problem for which CPET is required; 2) choose the exercise protocol; 3) ensure high quality results; 4) present results using a proper format; $5)$ select adequate reference values to establish patterns of abnormal response; and 6) compare with characteristic patterns of certain diseases.

In this section on interpretation of clinically oriented CPET, we first recommend a logical strategy to approach the problem. Comparative analysis of different sets of available reference values is also provided and, finally, detailed information on the variables indicated in table 5 (definition, information content, units and calculations) is described in the Appendix.

\section{General guidelines}

Several points should be considered in the interpretation of CPET results. First, suggestions in the literature supporting interpretative strategies based on single key measurements to direct the flow-chart decision did not prove to be adequate. The greatest diagnostic potential and impact on the clinical decision making process

Table 5. - Important variables and recommended plots

\begin{tabular}{ll}
\hline Category & Variables \\
\hline Mechanical work & Work rate (W) \\
Gas exchange & $V^{\prime} \mathrm{O}_{2}, V^{\prime} \mathrm{CO}_{2}, \mathrm{RER}, \mathrm{LT}$ \\
Ventilation & $V^{\prime} \mathrm{E}, V \mathrm{~T}, f, \mathrm{VR}$ \\
Respiratory blood gases & $\mathrm{Pa}_{\mathrm{a}, \mathrm{O}_{2}, P \mathrm{a}, \mathrm{CO}_{2}, \mathrm{DA}-\mathrm{a}, \mathrm{O}_{2}, V \mathrm{D} / V \mathrm{~T}, \mathrm{~S}_{\mathrm{a}, \mathrm{O}_{2}}}$ \\
Cardiovascular & $f \mathrm{C}, \mathrm{HRR}, P_{\mathrm{a}, \text { sys }}, \mathrm{ECG}, \mathrm{O}_{2}$ pulse \\
Acid-base status & pHa, $P \mathrm{a}, \mathrm{CO}_{2}$, base excess or \\
& standard $\mathrm{CO}_{3} \mathrm{H}^{-}$concentration \\
Symptoms & Dyspnoea, leg pain, chest pain \\
\hline
\end{tabular}

Basic plots

$V^{\prime} \mathrm{CO}_{2}$ (ordinate) $v s V^{\prime} \mathrm{O}_{2}$ (abscissa)

$V^{\prime} \mathrm{E} / V^{\prime} \mathrm{CO}_{2}$ (ordinate) and $V^{\prime} \mathrm{E} / V^{\prime} \mathrm{O}_{2}$ (ordinate) $v s V^{\prime} \mathrm{O}_{2}$ (abscissa)

$P$ ET, $\mathrm{CO}_{2}$ (ordinate) and $P$ ET, $\mathrm{O}_{2}$ (ordinate) $v s V^{\prime} \mathrm{O}_{2}$ (abscissa)

RER (ordinate) $v s V^{\prime} \mathrm{O}_{2}$ (abscissa)

$V^{\prime} \mathrm{O}_{2}$ (ordinate) $v s$ work rate (abscissa)

$V^{\prime}$ E (ordinate) $v s V^{\prime} \mathrm{CO}_{2}$ (abscissa) or $V^{\prime} \mathrm{E}$ (ordinate) $v s V^{\prime} \mathrm{O}_{2}$ (abscissa)

$f \mathrm{C}$ (ordinate) and $V^{\prime} \mathrm{CO}_{2} / f \mathrm{C}$ (ordinate) $v s V^{\prime} \mathrm{O}_{2}$ (abscissa)

$V$ T (ordinate) $v s V^{\prime} \mathrm{E}$ (abscissa)

Examples of the basic plots are shown in figure $7 . V^{\prime} \mathrm{O}_{2}$ : oxygen consumption; $V^{\prime} \mathrm{CO}_{2}$ : carbon dioxide production; RER: respiratory exchange ratio; LT: lactate threshold; VR: ventilatory reserve; $f$ : frequency; $P \mathrm{a}, \mathrm{O}_{2}$ : arterial oxygen tension; DA-a, $\mathrm{O}_{2}$ : alveolar-arterial oxygen difference; $V \mathrm{D}$ : dead space volume; $V$ T: tidal volume; $\mathrm{Sa}_{\mathrm{a}} \mathrm{O}_{2}$ : arterial oxygen saturation; $f \mathrm{C}$ : cardiac frequency; HRR: heart rate reserve; $P$ a,sys: systemic arterial pressure; ECG: electrocardiogram; pHa: arterial $\mathrm{pH} ; P_{\mathrm{a}, \mathrm{CO}_{2}}$ : arterial carbon dioxide tension; $V^{\prime} \mathrm{E}$ : minute ventilation; $P \mathrm{ET}, \mathrm{CO}_{2}$ : end tidal partial pressure of $\mathrm{CO}_{2} ; P \mathrm{ET}, \mathrm{O}_{2}$ : end tidal partial pressure of $\mathrm{O}_{2}$. should rely not on the utility of any one individual measurement, although some are obviously more important than others, but rather on their integrated use [1]. Consequently, a key point is the identification of a cluster of responses characteristic of different diseases.

The major portion of the interpretation strategy is focused on CPET results generated during maximal, symptom-limited, incremental exercise testing. This is currently the most popular, albeit not exclusive, protocol. Often, insufficient attention is paid to trending phenomena as the work rate progresses from submaximal to peak levels. To facilitate this type of analysis, the results should be formatted in an appropriate manner. While various display formats can be helpful in this regard (see [2] for example), one suggested format is presented in figure 7 , which displays data obtained in a normal subject performing cycle ergometry, using an ergometer that utilizes an "assist" to provide an actual $0 \mathrm{~W}$ work rate at "unloaded" pedalling. Figures $7 \mathrm{a}-\mathrm{d}$ provide, in addition to the peak $V^{\prime} \mathrm{O}_{2}$, the variables commonly used to provide an indirect estimation of the LT (as described in the Appendix using a cluster of relevant responses for the estimation, rather than relying on a single index). That is, the $V^{\prime} \mathrm{O}_{2}$ at which the transition between moderate and heavy-intensity exercise occurs is identified. Figure $7 \mathrm{e}\left(V^{\prime} \mathrm{O}_{2}\right.$ versus work rate) reflects the exercise efficiency (see section on Response to exercise in lung disease, subsection on Pulmonary gas exchange) and the limits of exercise tolerance of the subject. Figure $7 \mathrm{f}$ (ventilation versus $V^{\prime} \mathrm{CO}_{2}$ ) and figure $7 \mathrm{~h}$ ( $V \mathrm{~T}$ versus ventilation) characterize aspects of the ventilatory response during submaximal and maximal exercise. However, some investigators find the relationship between $V^{\prime} \mathrm{E}$ and $V^{\prime} \mathrm{O}_{2}$ during such tests to be useful. Finally, figure $7 \mathrm{~g}$ is informative with respect to the characteristics of the haemodynamic response to exercise. The next step is to choose adequate reference values to establish patterns of normal or abnormal response. Available data on reference values and present limitations in this particular issue are discussed below. This graphical analysis (fig. 7) can be even more illustrative of the subject's exercise performance if the corresponding reference values are also displayed in each plot (see [2] for example). A proper interpretation of CPET results should rely on both numerical analysis of the variables of interest (table 5) and assessment of trending phenomena throughout the exercise test using the graphical analysis suggested above. Comprehensive CPET can provide extremely useful information in the judgement on the system's tolerance to exercise by helping to answer the following basic questions in a given patient: 1) to what extent is the system constrained or limited? 2) how is it perceived? 3) are the metabolic requirements for a given task met? and 4) what is the cost of meeting the requirements?

The final step in this approach to the interpretation of CPET results is to identify clusters of responses characteristic of different diseases. It should be pointed out that each step of the proposed interpretative strategy is important; unless a flow-chart logic (e.g. [2]) is followed, the analysis of CPET results is prone to misinterpretations. Different algorithms to guide the decision making process, in discriminating a range of functional abnormalities, have been reported $[1,2,87]$ and are 

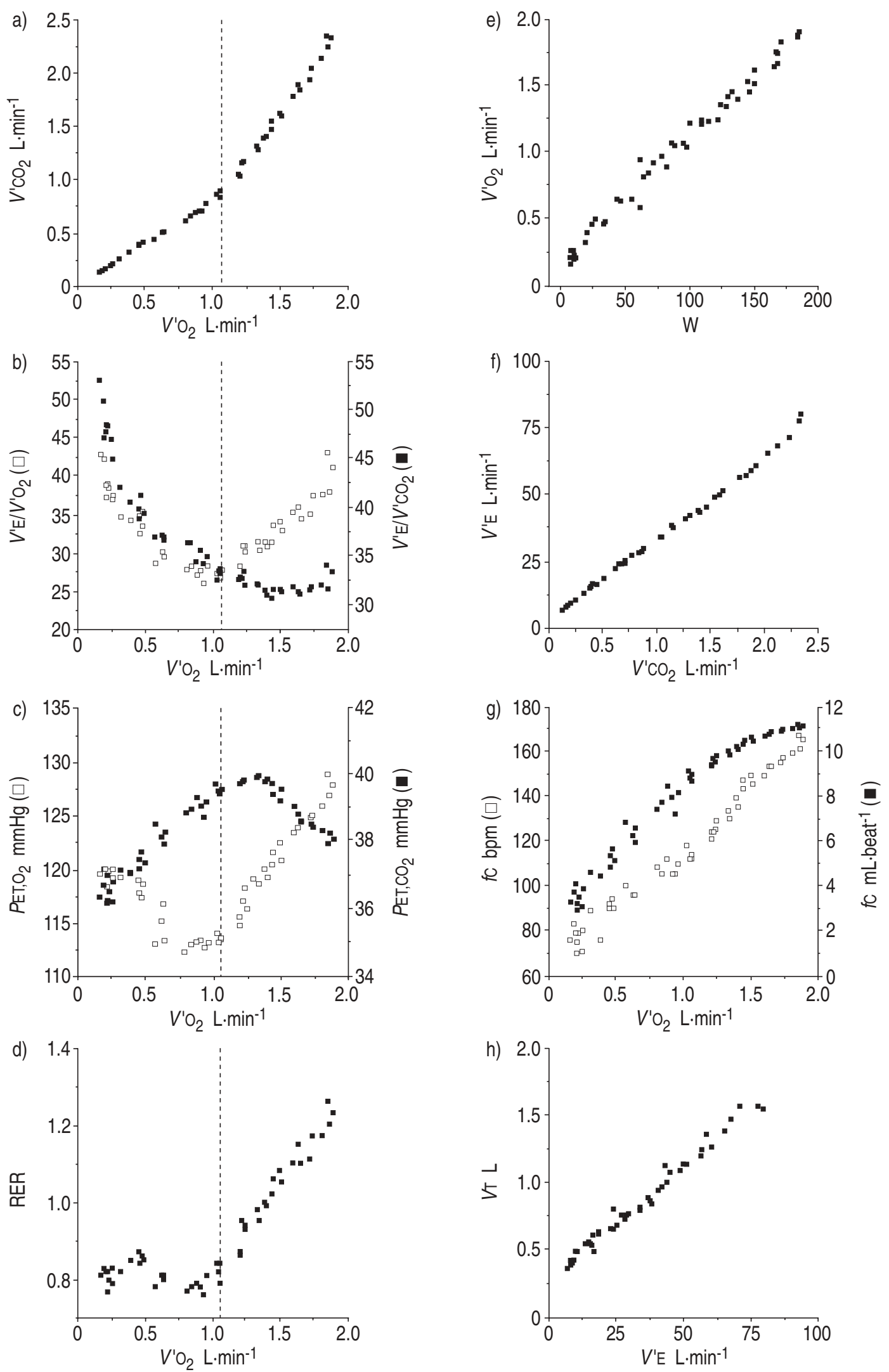

Fig. 7. - Basic plots recommended for the interpretation of CPET. Figures a-d, in addition to peak $V^{\prime} \mathrm{O}_{2}$, provide the variables commonly used to indirectly estimate the LT (as described in the appendix). That is, the $V^{\prime} \mathrm{O}_{2}$ at which the transition between moderate and heavy-intensity exercise occurs is identified. Figure e $\left(V^{\prime} \mathrm{O}_{2}\right.$ versus work rate) reflects the exercise efficiency and limits of exercise tolerance of the subject. Figure $\mathrm{f}$ (ventilation versus $V^{\prime} \mathrm{CO}_{2}$ ) and figure $\mathrm{h}$ (VT versus ventilation) characterize aspects of the ventilatory response during submaximal and maximal exercise. Finally, figure $\mathrm{g}$ is informative with respect to the characteristics of the haemodynamic response to exercise. For definitions see legend to table 5 . 
available in the literature. The logical flow commonly begins with the judgement of whether exercise tolerance was normal or not. If the subject shows abnormal limitation to exercise, the next logical step might be the analysis of the submaximal exercise region in order to identify the LT. If the physiological changes indicating the transition from moderate to heavy-intensity exercise occur within the lower end of the expected range of $V^{\prime} \mathrm{O}_{2}$ for a normal subject (but within it), then the dilemma is whether the subject had voluntarily stopped the test early or whether a physiological limitation to exercise performance can be identified. For example, were the analysis of the ventilatory function to show that the ventilatory reserve (VR; see Appendix) was zero or low but the heart rate reserve (HRR) was high at the end of the test, this suggests that the patient probably had a ventilatory limitation to the exercise, rather than stopping deliberately or as a result of acute discomfort, such as that of angina.

The above recommended guidelines for CPET interpretation propose a general logic framework for the analysis of limitation of exercise tolerance, e.g: cardiovascular (heart, systemic circulation and blood); respiratory (ventilation, pulmonary circulation and gas exchange); and peripheral muscle factors (neuromuscular-related abnormalities, impairment of muscle microvasculature, abnormal cellular oxygen or other substrate utilization). However, additional factors, such as psychogenic ones, can also be limiting. However, results of CPET, like other physiological tests in cardiopulmonary medicine, demonstrate patterns of abnormality with overlap between the responses of patients with different disorders. Table 6 provides a description of the most characteristic features of abnormal responses in different patient categories. In each of these categories there is one or more variables that have a predominant role in determining the severity of impairment. An expanded and detailed description of these patterns of abnormality can be found in elsewhere [85].

Relatively few studies have evaluated the sensitivity, specificity and predictive value of patterns of measurements in distinguishing different clinical entities. Even more importantly, the precise role of clusters of variables commonly used in the decision making process in well identified diseases (i.e., evaluation of ILD, preoperative evaluation for resectional lung cancer surgery, etc.) is insufficiently known. For the future, studies addressing the use of likelihood ratios [179] might be even more useful to clinicians than sensitivity and specificity, since likelihood ratios refer to actual test results before disease status is known. This shift to an evidencebased approach [180] for CPET interpretation will, hopefully, provide important answers to clinically relevant questions that are not immediately available.

\section{Reference values}

As indicated above, selection of appropriate reference values is an important step to establish patterns of normal or abnormal response to exercise stress. An initial analysis of available data on healthy subjects $[1,2,12$, $118,181-191]$ clearly indicated that only five of these studies [2, 181, 184-186] fulfill minimum requirements to be considered as candidates to be used in the clinical setting [85]. However, BLACKIE et al. [184] cover a limited age span (55-80 yrs), and BRUCE et al. [186] provide data obtained with treadmill in a population of physically fit people. Hence, the analysis of potential studies in healthy sedentary people providing prediction equations for peak $V^{\prime} \mathrm{O}_{2}$ obtained with incremental cycling exercise testing is reduced to three sets $[12,181$, 185]. Basic characteristics of these three studies are summarized in table 7 . Reference values estimated by FAIRBARN et al. [185] are consistently higher than those provided by JONES et al. [181]], both in men and women. The predicted values of HANSEN et al. [2, 12] are closer to either JoNEs et al. [181] or FAIRBARN et al. [185] depending upon the values of height-weight of the subject in whom the equations are used. The limitations of the presently available prediction equations for peak $V^{\prime} \mathrm{O}_{2}$ (and peak work rate) clearly impose limitations on the interpretative strategy. Moreover, except for $f \mathrm{C}$ in the study of FAIRBARN et al. [185], the profile of response in healthy sedentary subjects (i.e., from submaximal to peak exercise results) are not available. Furthermore, adequate prediction equations for even the most important variables (i.e., these indicated in table 5) obtained

Table 6. - Patterns of abnormal response to exercise in different diseases

\begin{tabular}{|c|c|c|c|c|c|c|}
\hline & COPD & ILD & PVD & Obesity & Deconditioned & Heart failure \\
\hline$V^{\prime} \mathrm{O}_{2}$,peak $(\%$ predicted $)$ & Reduced & Reduced & Reduced & $\begin{array}{l}\text { Reduced } \\
\text { Normal for } \\
\text { ideal weight }\end{array}$ & Reduced & Reduced \\
\hline $\mathrm{LT}$ & $\begin{array}{l}\text { Indeterminate, } \\
\text { normal or low }\end{array}$ & $\begin{array}{l}\text { Normal } \\
\text { or low }\end{array}$ & Low & Low & Normal or low & Low \\
\hline$V^{\prime}$ E,reserve & $\begin{array}{l}\text { Reduced or } \\
\text { none }\end{array}$ & $\begin{array}{l}\text { Normal or } \\
\text { reduced }\end{array}$ & Normal & Normal & Normal & Normal \\
\hline HRR & $\begin{array}{l}\text { Normal } \\
\text { or increased }\end{array}$ & $\begin{array}{l}\text { Normal } \\
\text { or increased }\end{array}$ & Normal & Normal & Normal & $\begin{array}{l}\text { Reduced } \\
\text { or normal }\end{array}$ \\
\hline $\mathrm{O}_{2}$ pulse at peak exercise & Reduced & Reduced & Reduced & Normal & Reduced & Reduced \\
\hline Fall in $\mathrm{Sa}, \mathrm{O}_{2}$ at peak exercise & $\begin{array}{l}\text { Present } \\
\text { or absent }\end{array}$ & Present & Present & Absent & Absent & Absent \\
\hline
\end{tabular}

COPD: chronic obstructive pulmonary disease; ILD: interstitial lung disease; PVD: pulmonary vascular obstructive disease; $V^{\prime} \mathrm{O}_{2}$, peak: oxygen uptake at peak exercise; LT: lactate threshold; $V$ 'E,reserve: see Appendix; HRR: heart rate reserve (see Appendix); $\mathrm{O}_{2}$ pulse: $V^{\prime} \mathrm{O}_{2}$, peak/peak cardiac frequency; $\mathrm{S}_{\mathrm{a}, \mathrm{O}_{2}}$ : oxyhaemoglobin saturation in arterial blood. 
Table 7. - Characteristics of the main sets of prediction equations

\begin{tabular}{|c|c|c|c|c|c|}
\hline First author [ref] & Gender & Equations & Population & Subjects & Equipment \\
\hline HANSEN $[2,12]$ & $\begin{array}{l}\mathrm{M} \\
\mathrm{F}\end{array}$ & $\begin{array}{l}V^{\prime} \mathrm{O}_{2}, \text { peak }\left(\mathrm{mL} \cdot \mathrm{min}^{-1}\right)(50.75-0.372 \mathrm{~A}) \mathrm{Wt} \\
V^{\prime} \mathrm{O}_{2}, \text { peak }\left(\mathrm{mL} \cdot \mathrm{min}^{-1}\right)(22.78-0.17 \mathrm{~A})(\mathrm{Wt}+43)\end{array}$ & $\begin{array}{c}77 \mathrm{M} \\
\text { Age } 34-74 \text { yrs }\end{array}$ & $\begin{array}{l}\text { Shipyard } \\
\text { workers }\end{array}$ & $\begin{array}{l}\text { Cycloergometer } \\
\text { Breath-by-breath } \\
10-30 \text { W increments }\end{array}$ \\
\hline JONES [181]* & $\begin{array}{l}M \\
F \\
M \\
F\end{array}$ & $\begin{array}{l}V^{\prime} \mathrm{O}_{2} \text {,peak }\left(\mathrm{L} \cdot \mathrm{min}^{-1}\right) 0.046 \mathrm{H}-0.021 \mathrm{~A}-4.31 \\
V^{\prime} \mathrm{O}_{2} \text {,peak }\left(\mathrm{L} \cdot \mathrm{min}^{-1}\right) 0.046 \mathrm{H}-0.021 \mathrm{~A}-4.93 \\
\mathrm{WR}\left(\mathrm{kpm} \cdot \mathrm{min}^{-1}\right) 20.4 \mathrm{H}-8.74 \mathrm{~A}-1909 \\
\mathrm{WR}\left(\mathrm{kpm} \cdot \mathrm{min}^{-1}\right) 20.4 \mathrm{H}-8.74 \mathrm{~A}-2197\end{array}$ & $\begin{array}{c}50 \mathrm{M} / 50 \mathrm{~F} \\
\text { Age } 15-71 \text { yrs }\end{array}$ & Volunteers & $\begin{array}{l}\text { Cycloergometer } \\
10 \mathrm{~L} \mathrm{mixing} \mathrm{box} \\
100 \mathrm{kpm} \cdot \mathrm{min}^{-1} \\
\text { increments }\end{array}$ \\
\hline BLACKIE [184] & $\begin{array}{l}\text { M } \\
\text { F } \\
\text { M } \\
\text { F }\end{array}$ & $\begin{array}{l}V^{\prime} \mathrm{O}_{2}, \text { peak }\left(\mathrm{L} \cdot \mathrm{min}^{-1}\right) 0.0142 \mathrm{H}-0.0494 \mathrm{~A} \\
+0.00257 \mathrm{Wt}+3.015 \\
V^{\prime} \mathrm{O}_{2}, \text { peak }\left(\mathrm{L} \cdot \mathrm{min}^{-1}\right) 0.0142 \mathrm{H}-0.0115 \mathrm{~A} \\
+0.00974 \mathrm{Wt}+0.651 \\
\mathrm{WR}\left(\mathrm{kpm} \cdot \mathrm{min}^{-1}\right) 6.1 \mathrm{H}-26.1 \mathrm{~A}+0.04 \mathrm{Wt}+1.704 \\
\mathrm{WR}\left(\mathrm{kpm} \cdot \mathrm{min}^{-1}\right) 7.4 \mathrm{H}-13.0 \mathrm{~A}+3.78 \mathrm{Wt}+52\end{array}$ & $\begin{array}{c}47 \mathrm{M} / 81 \mathrm{~F} \\
\mathrm{Age}>55 \mathrm{yrs}\end{array}$ & Volunteers & $\begin{array}{l}\text { Cycloergometer } \\
10 \mathrm{~L} \mathrm{mixing} \mathrm{box} \\
100 \mathrm{kpm} \cdot \mathrm{min}^{-1} \\
\text { increments }\end{array}$ \\
\hline FAIRBARN [185] & M & $\begin{array}{l}V^{\prime} \mathrm{O}_{2} \text {,peak }\left(\mathrm{L} \cdot \mathrm{min}^{-1}\right) 0.023 \mathrm{H}-0.031 \mathrm{~A} \\
+0.0117 \mathrm{Wt}-0.332 \\
V^{\prime} \mathrm{O}_{2} \text {,peak }\left(\mathrm{L} \cdot \mathrm{min}^{-1}\right) 0.0158 \mathrm{H}-0.027 \mathrm{~A} \\
+0.00899 \mathrm{Wt}+0.207\end{array}$ & $\begin{array}{l}111 \mathrm{M} / 120 \mathrm{~F} \\
\text { Age } 20-80 \text { yrs }\end{array}$ & Volunteers & $\begin{array}{l}\text { Cycloergometer } \\
8.5 \mathrm{~L} \mathrm{mixing} \mathrm{box} \\
100 \mathrm{kpm} \cdot \mathrm{min}^{-1} \\
\text { increments }\end{array}$ \\
\hline
\end{tabular}

M: male; F: female; A: age in years; H: height in centimetres; Wt: weight in kilograms; WR: work rate; kpm: kilopondmeters. For further definitions see legend to table 6 . HANSEN et al. [2, 12] suggest alternative equations for those subjects with differences between actual and ideal body weight. *: equations simplified from, Jones et al. [181] provide prediction equations for cardiac frequency and $\mathrm{O}_{2}$ pulse at peak exercise; FAIRBARN et al. [185] provide equations for cardiac frequency during submaximal and peak exercise.

Table 8. - Traditional versus SI units

\begin{tabular}{|c|c|c|c|c|}
\hline \multicolumn{2}{|c|}{$\begin{array}{l}\text { Traditional } \\
\text { units }\end{array}$} & \multicolumn{2}{|c|}{$\begin{array}{c}\text { SI } \\
\text { units }\end{array}$} & \multirow{2}{*}{ 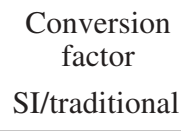 } \\
\hline Name & Units & Name & Units & \\
\hline \multicolumn{5}{|l|}{ Mechanical work } \\
\hline Work rate & $\begin{array}{l}\mathrm{kg} \cdot \mathrm{m} \cdot \mathrm{min}^{-1} \\
\text { or } \mathrm{kpm} \cdot \mathrm{min}^{-1}\end{array}$ & Watts & W & 0.163 \\
\hline \multicolumn{5}{|l|}{ Gas exchange } \\
\hline$V^{\prime} \mathrm{O}_{2}$ & $\mathrm{~mL} \cdot \mathrm{min}^{-1}$ & $n^{\prime} \mathrm{O}_{2}$ & $\mathrm{mmol} \cdot \mathrm{min}^{-1}$ & $22.40^{-1}$ \\
\hline$V^{\prime} \mathrm{CO}_{2}$ & $\mathrm{~mL} \cdot \mathrm{min}^{-1}$ & $n^{\prime} \mathrm{CO}_{2}$ & $\mathrm{mmol} \cdot \mathrm{min}^{-1}$ & $22.46^{-1}$ \\
\hline RER ${ }^{2}$ & - & RER & - & 1.0 \\
\hline \multicolumn{5}{|l|}{ Ventilation } \\
\hline$V^{\prime} \mathrm{E}$ & $\mathrm{L} \cdot \mathrm{min}^{-1}$ & $V^{\prime} \mathrm{E}$ & $\mathrm{L} \cdot \mathrm{min}^{-1}$ & 1.0 \\
\hline$V_{\mathrm{T}}$ & $\mathrm{mL}$ or $\mathrm{L}$ & $V \mathrm{~T}$ & $\mathrm{~mL}$ or $\mathrm{L}$ & 1.0 \\
\hline$f$ & breaths $\cdot \min ^{-1}$ & $f$ & breaths $\cdot \mathrm{min}^{-1}$ & 1.0 \\
\hline VR & - & VR & - & 1.0 \\
\hline \multicolumn{5}{|c|}{ Respiratory blood gases } \\
\hline $\mathrm{Pa}_{\mathrm{a}} \mathrm{O}_{2}$ & $\mathrm{mmHg}$ & $\mathrm{Pa}_{\mathrm{a}, \mathrm{O}_{2}}$ & $\mathrm{kPa}$ & 0.133 \\
\hline $\mathrm{Pa}_{\mathrm{a}, \mathrm{CO}_{2}}$ & $\mathrm{mmHg}$ & $\mathrm{Pa}_{\mathrm{a}, \mathrm{CO}_{2}}$ & $\mathrm{kPa}$ & 0.133 \\
\hline $\mathrm{DA}-\mathrm{a}, \mathrm{O}_{2}$ & $\mathrm{mmHg}$ & $\mathrm{DA}-\mathrm{a}, \mathrm{O}_{2}$ & $\mathrm{kPa}$ & 0.133 \\
\hline$V \mathrm{D} / V \mathrm{~T}$ & - & $V \mathrm{D} / V \mathrm{~T}$ & - & 1.0 \\
\hline \multicolumn{5}{|l|}{ Cardiovascular } \\
\hline$f \mathrm{C}$ & beats $\cdot \min ^{-1}$ & $f \mathrm{C}$ & beats $\cdot \min ^{-1}$ & 1.0 \\
\hline HRR & - & HRR & - & 1.0 \\
\hline$V^{\prime} \mathrm{O}_{2} / f \mathrm{C}$ & $\mathrm{mL}$ & $n^{\prime} \mathrm{O}_{2} / f \mathrm{C}$ & mmol & $22.4^{-1}$ \\
\hline \multicolumn{5}{|l|}{ Acid-base status } \\
\hline $\begin{array}{l}\text { Base excess } \\
\text { or bicarbonate }\end{array}$ & $\mathrm{mEq} \cdot \mathrm{L}^{-1}$ & $\begin{array}{l}\text { Base } \\
\text { excess }\end{array}$ & $\mathrm{mM}$ & 1.0 \\
\hline $\begin{array}{l}\text { Lactate } \\
\text { concentration }\end{array}$ & $\mathrm{mEq} \cdot \mathrm{L}^{-1}$ & $\begin{array}{l}\text { Lactate } \\
\text { concentration }\end{array}$ & $\mathrm{mM}$ & 1.0 \\
\hline
\end{tabular}

For definition of abbreviations see legend to table 5. $n^{\prime} \mathrm{O}_{2}, n^{\prime} \mathrm{CO}_{2}$ : millimoles per minute of oxygen and carbon dioxide, respectively; kpm: kilopondmeters; Conversion factor: Traditional unit to SI unit (SI/traditional unit).

from the same group of reference subjects are not currently available. The absence of appropriate reference values can naturally lead to miscategorization and possible misinterpretation of the results.

The Task Force members consider that an important goal in this area is to stimulate a multicentre study, with the aim of generating an appropriate set of reference values. This would establish both the magnitude and profiles of response of the essential CEPT variables utilizing standardized procedures and rigorous quality control (within and among participating laboratories). Additional potential gains from such a programme would be the comparison among incremental cycling, incremental treadmill and constant work load exercise testing in a subset of subjects. Such a project would provide a major advance in refining the ability to interpret CPET.

\section{Appendix}

\section{SI units}

SI stands for "Système International d'Unités" [192, 193]. These units are the result of over a century of international co-operation to develop a universally acceptable system of units of measurement. The SI is an outgrowth of the metric system that is being increasingly used all over the world, but which has had rather little impact in the United States of America (USA), even though Congress passed the Metric Conversion Act in 1975, which endorsed the SI scale. Formally, the SI units were adopted by all the members of the European Union about 20 yrs ago. Table 8 indicates both nomenclature and conversion factors of the SI units for the variables used in the present document. 
Variables: definitions and calculations

Mechanical work. Work rate (W). This indicates the amount of work performed per unit of time. Work $(\mathrm{kg}$. $\left.\mathrm{m}^{2} \cdot \mathrm{s}^{-2}, \mathrm{~J}\right)$, in turn, is a physical quantification of the force $\left(\mathrm{kg} \cdot \mathrm{m}^{2} \cdot \mathrm{s}^{-2}, \mathrm{~N}\right)$ operating on a mass $(\mathrm{kg})$ that causes its change of position (distance expressed in meters). The work rate (or power) is measured in watts $\left(\mathrm{kg} \cdot \mathrm{m}^{2}\right.$. $\mathrm{s}^{-3}$ or $\left.\mathrm{J} \cdot \mathrm{s}^{-1}\right)$.

Gas exchange. Oxygen uptake $\left(V^{\prime} \mathrm{O}_{2}\right.$, in $\mathrm{mL} \cdot \mathrm{min}^{-1}$ or $\left.\mathrm{L} \cdot \mathrm{min}^{-1}\right)$. Is the difference in oxygen flow between inspired and expired gas (in STPD conditions, see below). During steady-state conditions, oxygen uptake and consumption (amount used by the body metabolism in a given period of time) are equivalent. $V^{\prime} \mathrm{O}_{2}$ may be calculated as:

$$
V^{\prime} \mathrm{O}_{2}=V^{\prime} \mathrm{I} \cdot F \mathrm{I}, \mathrm{O}_{2}-V^{\prime} \mathrm{E} \cdot F \mathrm{E}, \mathrm{O}_{2}
$$

or, if only $V^{\prime} \mathrm{E}$ is determined:

$$
V^{\prime} \mathrm{O}_{2}=\left(F \mathrm{I}, \mathrm{O}_{2} \cdot\left(\frac{\mathrm{FE}, \mathrm{N}_{2}}{\mathrm{FI}, \mathrm{N}_{2}}\right)-F \mathrm{E}, \mathrm{O}_{2}\right) \cdot V^{\prime} \mathrm{E}
$$

where $V^{\prime} \mathrm{E}$ is expiratory minute ventilation and $V^{\prime} \mathrm{I}$ is inspiratory minute ventilation, $F \mathrm{I}, \mathrm{O}_{2}$ and $\mathrm{FE}, \mathrm{O}_{2}$ are the fractional concentrations of inspired oxygen and expired oxygen, and FI, $\mathrm{N}_{2}$ and FE, $\mathrm{N}_{2}$ are the fractional concentrations of inspired and expired nitrogen.

Carbon dioxide output $\left(V^{\prime} \mathrm{CO}_{2} \mathrm{~mL} \cdot \mathrm{min}^{-1}\right)$. In the absence of inspired carbon dioxide, $V^{\prime} \mathrm{CO}_{2}$ is the flow of carbon dioxide exhaled from the body into the atmosphere, expressed in STPD conditions (see below). During steady state conditions, carbon dioxide output equals production by the body. Calculation of $V^{\prime} \mathrm{CO}_{2}$ can be computed as the total volume of carbon dioxide exhaled in a given period of time:

$$
V^{\prime} \mathrm{CO}_{2}=F \mathrm{E}, \mathrm{CO}_{2} \cdot V^{\prime} \mathrm{E}
$$

where $\mathrm{FE}, \mathrm{CO}_{2}$ is the fractional concentration of expired carbon dioxide.

Respiratory exchange ratio (RER or R, dimensionless). This is the ratio of $V^{\prime} \mathrm{CO}_{2}$ to $V^{\prime} \mathrm{O}_{2}$. RER reflects not only tissue metabolic activity, but also the influence of transient changes in body stores of respiratory gases (oxygen and, most importantly, carbon dioxide). The respiratory quotient (RQ) is the ratio of $V^{\prime} \mathrm{CO}_{2}$ to $V^{\prime} \mathrm{O}_{2}$ and reflects the metabolic substrate utilization. During hyperventilation, RER exceeds RQ because additional carbon dioxide from the body stores is exhaled, whereas the RER is less than the RQ during transient hypoventilation when carbon dioxide is retained in the body stores.

Lactate threshold $\left(\mathrm{LT}, \mathrm{mL} \cdot \mathrm{min}^{-1}\right)$. This is the exercise $V^{\prime} \mathrm{O}_{2}$ above which a net increase in lactate production results in a sustained increase in blood lactate concentration. The LT provides: 1) an index of the functional status of the respiratory-circulatory-metabolic integration that allows exercise to be sustained aerobically; 2) an index of sustainability for that particular task; 3) a frame of reference for optimizing training protocols and monitoring physical training, rehabilitation and drug in- terventions; and 4) a component of decision-making strategies for elucidating the dominant system(s) responsible for exertional dyspnoea, and exercise intolerance. Its limitations, however, in patients with lung disease have been indicated in the text and are further analysed below:

1) Direct measurements of LT. The response profile of arterial lactate concentration versus $V^{\prime} \mathrm{O}_{2}$ is often not the most sensitive estimator for LT, as there may be no clear "break-point" in the profile that can be identified with sufficient confidence (for some investigators, at least).

2) Arterial lactate-pyruvate concentration ratio. As a component of the arterial lactate concentration, increase reflects pyruvate-dependent increase, as a result of increased glycolytic flux; the ratio of arterial lactate to pyruvate concentrations provides a clearer manifestation of the threshold behaviour.

3) Logarithmic arterial lactate and bicarbonate concentration functions. This approach transforms the arterial lactate concentration (or arterial standard bicarbonate concentration, standard $\left.\left(\left[\mathrm{HCO}_{3}^{-}\right]\right)\right)$response to a logarithmic function. This provides an even clearer discrimination of the lower (i.e. subthreshold) and higher (i.e. suprathreshold) regions of the response. It typically linearizes the more sharply rising phase of the response, intersecting the lower phase of the response, which commonly displays a small positive slope. It is important, however, that the appropriate sampling site is used for determining LT. Arterial (or mixed venous, in some instances) blood is most appropriate. Properly "arterialized" venous blood is acceptable.

Noninvasive estimators of LT (ventilatory equivalent method and V-slope method) [194, 195] are described below. As indicated previously (see section on Equipment and quality control programme), the discriminability of LT can be enhanced in some cases by utilizing a particular cluster of ventilatory and pulmonary gas exchange responses.

Ventilatory equivalent method. The compensatory hyperventilation for the metabolic acidosis of heavy and severe exercise typically coincides with the increase in arterial lactate concentration and the decrease in arterial standard $\left[\mathrm{HCO}_{3}^{-}\right]$. As a test for this, the work rate is incremented in a quasi-steady state manner (i.e. increment duration of 3-4 min or more); $V^{\prime} \mathrm{E}$ begins to increase more rapidly than $V^{\prime} \mathrm{CO}_{2}$ and $V^{\prime} \mathrm{O}_{2}$ (i.e. the ventilatory equivalents for oxygen and carbon dioxide $\left(V^{\prime} \mathrm{E} / V^{\prime} \mathrm{O}_{2}, V^{\prime} \mathrm{E} /\right.$ $\left.V^{\prime} \mathrm{CO}_{2}\right)$ both increase and $P \mathrm{a}, \mathrm{CO}_{2}$ is, therefore, reduced.

In contrast, however, when the work rate incrementation rate is more rapid, the compensatory hyperventilation is strikingly attenuated. In this situation, there is a range of work rates immediately above LT within which $V^{\prime} \mathrm{E}$ and $V^{\prime} \mathrm{CO}_{2}$ increase in approximately the same proportionality as for moderate exercise; i.e. $V^{\prime} \mathrm{E} / V^{\prime} \mathrm{CO}_{2}$ does not increase. Under these conditions, $V^{\prime} \mathrm{CO}_{2}$ has contributions both from metabolic sources and from bicarbonate buffering reactions. Therefore, as $P_{\mathrm{a}}, \mathrm{CO}_{2}$ does not fall in this region, it has been termed the range of "isocapnic buffering". However, the additional $V^{\prime} \mathrm{E}$ that clears this augmented load of carbon dioxide is, therefore, out of proportion to $V^{\prime} \mathrm{O}_{2}$ causing $V^{\prime} \mathrm{E} / V^{\prime} \mathrm{O}_{2}$ to increase. Respiratory compensation for the lactic acidosis 
(i.e. with $P \mathrm{a}, \mathrm{CO}_{2}$ actually decreasing) only begins for rapid incremental tests at a work rate which is typically about midway between LT and $V^{\prime} \mathrm{O}_{2}$, max. This has been termed the "respiratory compensation point". It is, therefore, important to recognize that the respiratory compensation point is a function of the incrementation rate of the test, whereas LT is not.

The phenomenon of isocapnic buffering consequently helps rule out nonspecific hyperventilation (such as is seen in some excitable subjects and also in patients with McArdle's syndrome) as a "false positive" lactate threshold. For an appropriate rapid incremental exercise protocol, LT has typically been taken as the $V^{\prime} \mathrm{O}_{2}$ (not the work rate) at which the alveolar (end-tidal) $\mathrm{PO}_{2}$ $\left(P \mathrm{ET}, \mathrm{O}_{2}\right)$ and the ventilatory equivalent for oxygen $\left(V^{\prime} \mathrm{E} /\right.$ $\left.V^{\prime} \mathrm{O}_{2}\right)$ start to rise systemically without a simultaneous fall in $P$ ET, $\mathrm{CO}_{2}$.

Some individuals (such as those with intensive peripheral chemoreceptors or those with appreciably increased airways resistance), may have a compromised ability to generate the required increases in $V^{\prime} \mathrm{E}$ at higher work rates. As a result, if there is no, or possibly little, additional ventilatory response, it proves difficult, or impossible, to discriminate LT according to these ventilatory-related criteria. The V-slope approach was developed to overcome this challenge to discrimination.

The V-slope method. With this procedure, LT is identified from the relationship between $V^{\prime} \mathrm{CO}_{2}$ and $V^{\prime} \mathrm{O}_{2}$. The acceleration of the rate of increase in $V^{\prime} \mathrm{CO}_{2}$ relative to $V^{\prime} \mathrm{O}_{2}$ above the LT provides evidence for an increased, but still essentially linear, slope $\left(\mathrm{S}_{2}\right.$, fig. 8), within the isocapnic buffering phase. The intersection of the two linear phases $\left(S_{1}\right.$ and $S_{2}$, fig. 8$)$ has been shown to agree closely with the beginning of the increase in arterial lactate concentration and arterial lactate/pyruvate concentration ratio and even more closely with the decrease in arterial standard $\left[\mathrm{CO}_{3} \mathrm{H}^{-}\right]$, i.e. a small amount of the initial increase in muscle lactate is buffered by nonbicarbonate buffers [2, 147], and hence does not yield increased $V^{\prime} \mathrm{CO}_{2}$. The consequence for LT discrimination, however, is likely to be disappearingly small during incremental testing. On those occasions, when the

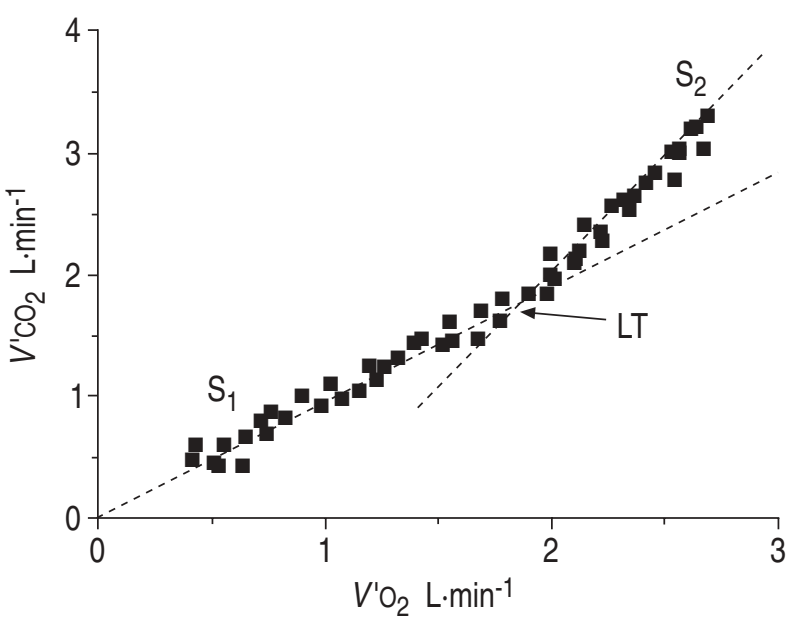

Fig. 8. - Estimation of lactate threshold (LT) using the V-slope method (see text for further explanations). The intersection of the slopes of the two linear phases $\left(S_{1}\right.$ and $\left.S_{2}\right)$ corresponds to the LT. For definitions, see legend to table 5 .
$S_{1}$ and $S_{2}$ regions are perceived not to be sufficiently linear for the intersection to be meaningful, it has been proposed that the point on the curve at which a line of slope 1.0 is tangential will provide an adequate approximation of the threshold [196].

Ventilation. Minute ventilation $\mathrm{L} \cdot \mathrm{min}^{-1}$. Volume of gas expired $\left(V^{\prime} \mathrm{E}\right)$ or inspired $\left(V^{\prime} \mathrm{I}\right)$ in one minute, expressed in BTPS conditions (see below).

Tidal volume ( $V \mathrm{~T}, \mathrm{~mL}$ or $\mathrm{L})$. Volume of gas inspired (or expired) during each breathing cycle. It is computed as the ratio of minute ventilation $\left(\mathrm{L} \cdot \mathrm{min}^{-1}\right)$ to respiratory frequency (breaths $\cdot \mathrm{min}^{-1}$ ).

Respiratory frequency $\left(f \mathrm{R}\right.$, in breaths $\left.\cdot \mathrm{min}^{-1}\right)$. Number of breathing cycles per minute. It is computed as the ratio 60 divided by the total time of the breathing cycle (s).

Ventilatory reserve (VR, dimensionless). Difference between ventilatory capacity during maximum exercise (estimated as maximum voluntary ventilation (MVV)) and minute ventilation at peak exercise, expressed as a percentage of MVV. It is computed as:

$$
\text { VR } \%=\left(\left(\mathrm{MVV}-\text { peak } V^{\prime} \mathrm{E}\right) / \mathrm{MVV}\right) \cdot 100
$$

It, thus, represents the potential for further increase in ventilation during maximal (or peak) exercise.

Respiratory blood gases. Arterial $\mathrm{PO}_{2}\left(\mathrm{~Pa}, \mathrm{O}_{2}, \mathrm{mmHg}\right)$. Oxygen partial pressure in arterial blood.

Arterial $\mathrm{PCO}_{2}\left(\mathrm{~Pa}, \mathrm{CO}_{2} \mathrm{mmHg}\right)$. Carbon dioxide partial pressure in arterial blood.

Alveolar-arterial oxygen difference (DA-a, $\mathrm{O}_{2}, \mathrm{mmHg}$ ). Difference between ideal alveolar partial pressure of oxygen $\left(P \mathrm{~A}, \mathrm{O}_{2}\right)$ and measured $\mathrm{Pa}, \mathrm{O}_{2}$. The DA-a, $\mathrm{O}_{2}$ is computed using the alveolar gas equation described in the opening section:

$$
\begin{aligned}
& \mathrm{DA}-\mathrm{a}, \mathrm{O}_{2}=\left(\mathrm{PI}, \mathrm{O}_{2}-P \mathrm{a}, \mathrm{CO}_{2} / \mathrm{RER}+\right. \\
& \left(\mathrm{Pa}, \mathrm{CO}_{2} \cdot \mathrm{FI}, \mathrm{O}_{2} \cdot(1-\mathrm{RER}) / \mathrm{RER}\right)-\mathrm{Pa}_{\mathrm{a}} \mathrm{O}_{2}
\end{aligned}
$$

where RER is the respiratory exchange ratio (RER= $\left.V^{\prime} \mathrm{CO}_{2} / V^{\prime} \mathrm{O}_{2}\right)$. This equation is derived from mass balance considerations of oxygen and carbon dioxide exchange between the air and the alveolar gas and assumes constancy of lung nitrogen stores. Thus, even if oxygen, carbon dioxide and $V^{\prime} \mathrm{E}$ are changing, the $\mathrm{DA}-\mathrm{a}, \mathrm{O}_{2}$ can be validly computed if there is nitrogen balance between inspiration and expiration. It is important to recognize that the absolute blood gas values and the $\mathrm{DA}-\mathrm{a}, \mathrm{O}_{2}$ yield different, but complementary information. The DA-a, $\mathrm{O}_{2}$ primarily reflects pulmonary defects in gas exchange caused by $V^{\prime} \mathrm{A} / Q^{\prime}$ mismatching, diffusion limitation and shunt, either alone or in combination. It can, however, be modified by changes in cardiac output or ventilation even in the absence of change in $V^{\prime} \mathrm{A} / Q^{\prime}$ distribution.

Dead space to tidal volume ratio $(V \mathrm{D} / V \mathrm{~T}$ dimensionless). Portion of tidal volume $(V \mathrm{~T})$ ventilating physiologic dead space $(V D)$. It is an index of the efficiency 
of the lung as carbon dioxide exchanger. It is computed as the ratio of the difference between $P \mathrm{a}, \mathrm{CO}_{2}$ and mixed expired $\mathrm{PCO}_{2}\left(\mathrm{~Pa}, \mathrm{CO}_{2}-P \mathrm{E}, \mathrm{CO}_{2}\right)$ to $P \mathrm{a}, \mathrm{CO}_{2}$ :

$$
\mathrm{VD} / \mathrm{VT} \%=\left(\left(P \mathrm{a}, \mathrm{CO}_{2}-P \mathrm{E}, \mathrm{CO}_{2}\right) / P \mathrm{a}, \mathrm{CO}_{2}\right) \cdot 100
$$

Cardiovascular. Cardiac frequency ( $f \mathrm{C}$, beats. $\left.\mathrm{min}^{-1}\right)$. Number of cardiac beats per minute. It is computed as the ratio of number of complete beats during $1 \mathrm{~min}$ to the total time of the cardiac cycle.

Heart rate reserve (HRR, dimensionless). Ratio of the difference between the predicted highest cardiac frequency at maximum exercise and the measured peak cardiac frequency to the predicted highest cardiac frequency at maximum exercise, expressed as a percentage.

$$
\begin{aligned}
\text { HRR }= & ((\text { Predicted maximum } f C-\text { peak } f C) / \\
& \text { predicted maximum } f C) \cdot 100
\end{aligned}
$$

$\mathrm{O}_{2}$ pulse $\left(V^{\prime} \mathrm{O}_{2} / f \mathrm{C}, \mathrm{mL}\right)$. Corresponds to the amount of oxygen uptake per cardiac cycle. The $\mathrm{O}_{2}$ pulse is numerically equal to the product of the stroke volume and the arterial-to-venous oxygen content difference. Consequently, inferences which are drawn about one of the contributory variables are highly dependent on assumptions regarding the response of the other(s).

Acid-base status. Arterial pH (pHa). Inverse of the decimal logarithm of the arterial blood hydrogen ion concentration $\left(\left[\mathrm{H}^{+}\right]\right)$. It indicates the global acid-base status (respiratory and metabolic components) of the arterial blood.

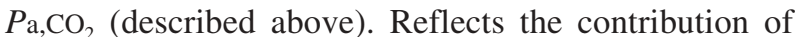
the respiratory component to the acid-base status.

Base excess $\left(\mathrm{BE}, \mathrm{mEq} \cdot \mathrm{L}^{-1}\right)$. Indicates the contribution of the metabolic component (nonvolatile substances) to the acid-base status of the blood. The BE is computed using the Henderson-Hasselbach equation with measured values of $\mathrm{pHa}$ and $\mathrm{Pa}_{2}, \mathrm{CO}_{2}$. Haemoglobin concentration and oxyhaemoglobin saturation influence the $\mathrm{BE}$ calculation by modulating the buffer capacity of the blood. The information provided by this variable is similar to that obtained estimating the standard $\left[-\mathrm{CO}_{3} \mathrm{H}\right]$.

Measurement conditions. STPD. Gas volume measurements at standard conditions of temperature $\left(0^{\circ} \mathrm{C}\right)$ and pressure $(760 \mathrm{mmHg}(101.3 \mathrm{kPa}))$, free of water vapour (partial pressure of water vapour $\mathrm{PH}_{2} \mathrm{O}=0 \mathrm{kPa}$ ).

BTPS. Gas volume measurements at body temperature $\left(37^{\circ} \mathrm{C}\right)$, ambient atmospheric pressure and saturated with water vapour at body temperature $\left(\mathrm{PH}_{2} \mathrm{O}=47 \mathrm{mmHg}\right.$ $(6.2 \mathrm{kPa}))$.

Acknowledgements: The members of the Task Force on Clinical Exercise Testing are grateful to M. Hillenius and P. Chapman for their skilful assistance in the preparation of the manuscript and to F. Burgos for preparing the figures.

\section{References}

1. Weisman IM, Zeballos RJ. An integrated approach to the interpretation of CPET. In: Weisman IM, Zeballos RJ, eds. Clinical Exercise Testing; Clinics in Chest Medicine. Philadelphia, W.B. Saunders, 1994.

2. Wasserman K, Hansen JE, Sue DY, Whipp BJ, Casaburi R. Principles of Exercise Testing and Interpretation. Philadelphia, Lea \& Febiger, 1994.

3. Killian KJ, Leblanc P, Martin DH, Summers E, Jones NL, Campbell EJM. Exercise capacity and ventilatory, circulatory, and symptom limitation in patients with chronic airflow limitation. Am Rev Respir Dis 1992; 146: 935-940.

4. Ortega F, Montemayor T, Sanchez A, Cabello F, Castillo J. Role of cardiopulmonary exercise testing and the criteria used to determine disability in patients with severe chronic obstructive pulmonary disease. Am J Respir Crit Care Med 1994; 150: 747-751.

5. Weisman IM, Zeballos RJ. Cardiopulmonary exercise testing. Pulmonary Critical Care Update series 1995; 11: 1-9.

6. Oren A, Sue DY, Hansen JE, Torrance DJ, Wasserman $\mathrm{K}$. The role of exercise testing in impairment evaluation. Am Rev Respir Dis 1987; 135: 230-235.

7. Cotes JE, Zejda J, King B. Lung function impairment as a guide to exercise limitation in work-related lung disorders. Am Rev Respir Dis 1988; 137: 1089-1093.

8. Sue DY. Exercise testing in the evaluation of impairment and disability. Clin Chest Med 1994; 15: 369-387.

9. Curtis JR, Deyo RA, Hudson LD. Health-related quality of life among patients with chronic obstructive pulmonary disease. Thorax 1994; 49: 162-170.

10. Knight DR, Schaffartzik W, Poole DC, Hogan MC, Bebout DE, Wagner PD. Effects of hyperoxia on maximal leg $\mathrm{O}_{2}$ supply and utilization in men. $J$ Appl Physiol 1993; 75: 2586-2594.

11. Belman MJ. Exercise in chronic obstructive pulmonary disease. In: Ryan AJ, Allman FLJ, eds. Sports Medicine. New York, Academic Press, 1989; pp. 647669.

12. Hansen JE, Sue DY, Wasserman K. Predicted values for clinical exercise testing. Am Rev Respir Dis 1984; 129: S49-S50.

13. Nery LE, Wasserman K, Andrews JD, Huntsman DJ, Hansen JE, Whipp BJ. Ventilatory and gas exchange kinetics during exercise in chronic airways obstruction. J Appl Physiol 1982; 53: 1594-1602.

14. Linnarsson D. Dynamics of pulmonary gas exchange and heart rate at start and end of exercise. Acta Physiol Scand 1974; 415: 1-68.

15. Whipp BJ. Dynamics of pulmonary gas exchange during exercise in man. Circulation 1987; 76 (6 part 2) VI: 18-28.

16. Dantzker DR, D'Alonzo GE. The effect of exercise on pulmonary gas exchange in patients with severe chronic obstructive pulmonary disease. Am Rev Respir Dis 1986; 134: 1135-1139.

17. Gallagher CG. Exercise limitation and clinical exercise testing in chronic obstructive pulmonary disease. Clin Chest Med 1994; 15: 305-326.

18. Agustí AG, Barberà JA, Roca J, Rodriguez-Roisin R, Wagner PD, Agustí-Vidal A. Hypoxic pulmonary vasoconstriction and gas exchange during exercise in chronic obstructive pulmonary disease. Chest 1990; 97 : 268-275.

19. Jones NL, Killian KJ. Exercise in chronic airway 
obstruction. In: Bouchard C, ed. Exercise Fitness and Health. Champaign, IL, USA. Human Kinetics Publishers, 1990; pp. 547-559.

20. Hansen JE. Respiratory abnormalities: exercise evaluation of the dyspnoeic patient. In: Leff AR, ed. Cardiopulmonary Exercise Testing. New York, Grune and Stratton, 1986; pp. 68-88.

21. Wagner PD, Gale GE. Ventilation-perfusion relationships. In: Whipp BJ, Wasserman K, eds. Pulmonary Physiology and Pathophysiology of Exercise. New York, Dekker, 1991; pp. 121-142.

22. D'Alonzo GE, Gianotti LA, Pohil RL. Comparison of progressive exercise performance of normal subjects and patients with primary pulmonary hypertension. Chest 1987; 92: 57-62.

23. Agustí AG, Roca J, Rodriguez-Roisin R, Gea J, Xaubet A, Wagner PD. Mechanisms of gas exchange impairment in idiopathic pulmonary fibrosis. Am Rev Respir Dis 1991; 143: 219-225.

24. Dantzker DR, D'Alonzo GE, Bower JS, Popat K, Crevey BJ. Pulmonary gas exchange during exercise in patients with chronic obliterative pulmonary hypertension. Am Rev Respir Dis 1984; 130: 412-416.

25. Jones NL, Rebuck AS. Tidal volume during exercise in patients with diffuse fibrosing alveolitis. Bull Eur Physiopathol Respir 1979; 15: 321-327.

26. Potter WA, Olafsson S, Hyat RE. Ventilatory mechanics and expiratory flow limitation during exercise in patients with obstructive lung disease. J Clin Invest 1971; 50: 910-919.

27. Gallagher C. Exercise and chronic obstructive pulmonary disease. Med Clin N Am 1990; 74: 619-641.

28. Johnson BD, Dempsey JA. Demand versus capacity in the aging pulmonary system. Ex Sports Sci Rev 1991; 19: 171-210.

29. Younes M. Determinants of thoracic excursions during exercise. In: Whipp BJ, Wasserman K, eds. Pulmonary Physiology and Pathophysiology of Exercise. New York, Dekker, 1991; pp. 1-65.

30. Marciniuk DD, Gallagher CG. Clinical exercise testing in interstitial lung disease. Clin Chest Med 1994; 15: 287-303.

31. Scano G, Gigliotti F, van Meerhaeghe A, et al. Influence of exercise and $\mathrm{CO}_{2}$ on breathing pattern in patients with chronic obstructive lung disease (COLD). Eur Respir J 1988; 1: 139-144.

32. Raimondi AC, Edwards RHT, Denison DM, et al. Exercise tolerance breathing a low density gas mixture, $35 \%$ oxygen and air in patients with chronic obstructive bronchitis. Clin Sci 1970; 39: 675-685.

33. Nattie EE, Tenney SM. The ventilatory response to resistance unloading during muscular exercise. Respir Physiol 1970; 20: 249-262.

34. Hussain SNA, Pardy RL, Dempsey JA. Mechanical impedance as determinant of inspiratory neural drive during exercise in humans. J Appl Physiol 1985; 59: 365-375.

35. Wagner PD, Dantzker DR, Dueck R, et al. Ventilationperfusion inequality in chronic obstructive pulmonary disease. J Clin Invest 1977; 59: 203-216.

36. Stewart RI, Lewis CM. Cardiac output during exercise in patients with COPD. Chest 1986; 89: 199-205.

37. Wagner PD, Hoppeler H, Saltin B. Determinants of maximal oxygen uptake. In: Crystal RG, West JB, eds. The Lung: Scientific Foundations. New York, Raven Press, 1991; pp. 1585-1594.

38. Wahren J, Felig P, Havel RJ, Jorfeldt L, Pernow B,
Saltin B. Amino acid metabolism in McArdle's syndrome. N Engl J Med 1973; 288: 774-777.

39. Martinez FJ, Stanopoulos I, Acero R, Becker FS, Pickering $\mathrm{R}$, Beamis JF. Graded comprehensive cardiopulmonary exercise testing in the evaluation of dyspnea unexplained by routine evaluation. Chest 1994; 105: 168-174.

40. Weisman IM, Zeballos RJ. Clinical evaluation of unexplained dyspnea. Cardiologia 1996; 41: 621-634.

41. Keogh BA, Lakatos E, Price D, et al. Importance of the lower respiratory tract in oxygen transfer: exercise testing in patients with interstitial and destructive lung disease. Am Rev Respir Dis 1984; 129: S76-S80.

42. Kelley MA, Daniele RP. Exercise testing in interstitial lung disease. Clin Chest Med 1984; 5: 145-156.

43. Fulmer JD, Roberts WC, Von Gal ER, Crystal RG. Morphologic-physiologic correlates of the severity of fibrosis and degree of cellularity in idiopathic pulmonary fibrosis. J Clin Invest 1979; 63: 665-676.

44. Agustí C, Xaubet A, Roca J, Agustí AG, RodriguezRoisin R. Interstitial pulmonary fibrosis with and without associated collagen vascular disease: results of a 2 years follow up. Thorax 1992; 47: 1035-1040.

45. Nickoladze GD. Functional results of surgery for bullous emphysema. Chest 1992; 101: 119-122.

46. Cooper JD, Trulock EP, Triantafillou AN, et al. Bilateral pneumectomy (volume reduction) for chronic obstructive pulmonary disease. J Thorac Cardiovasc Surg 1995; 109: 106-119.

47. D'Alonzo GE, Gianotti L, Dantzker DR. Noninvasive assessment of haemodynamic improvement during chronic vasodilator therapy in obliterative pulmonary hypertension. Am Rev Respir Dis 1986; 133: 380-384.

48. Biggar DG, Malen JF, Trulock EP, Cooper JD. Pulmonary rehabilitation before and after lung transplantation. In: Casaburi R, Petty TL, eds. Principles and Practice of Pulmonary Rehabilitation. Philadelphia, W.B. Saunders Co., 1993; pp. 459-467.

49. Rubin LJ. ACCP Consensus Statement. Primary pulmonary hypertension. Chest 1993; 104: 236-250.

50. Rhodes J, Barst RJ, Garofano RP, Thoele DG, Gersony WM. Haemodynamic correlates of exercise function in patients with primary pulmonary hypertension. $J \mathrm{Am}$ Coll Cardiol 1991; 18: 1738-1744.

51. Nixon PA, Orenstein DM, Kelsey SF, Doershuk CF. The prognostic value of exercise testing in patients with cystic fibrosis. N Engl J Med 1992; 327: 1785-1788.

52. Older P, Hall A. The role of cardiopulmonary exercise testing for preoperative evaluation of the elderly. In: Wasserman K, ed. Exercise Gas Exchange in Heart Disease. New York, Armonk: Futura Publishing Company, 1996; Chapter 20.

53. Shoemaker WC, Appel PL, Kram HB. Role of oxygen debt in the development of organ failure sepsis, and death in high-risk surgical patients. Chest 1992; 102: 208-215.

54. Marshall MC, Olsen GN. The physiologic evaluation of the lung resection candidate. Clin Chest Med 1993; 14: $305-320$.

55. Ferguson MK, Little L, Rizzo L, et al. Diffusing capacity predicts morbidity and mortality after pulmonary resection. J Thorac Cardiovasc Surg 1988; 96: 894-900.

56. Kearney DJ, Lee TH, Reilly JJ, DeCamp MM, Sugarbaker DJ. Assessment of operative risk in patients undergoing lung resection: importance of predicted pulmonary function. Chest 1994; 105: 753-759.

57. Markos J, Mullan BP, Hillman DR, et al. Preoperative assessment as a predictor of mortality and morbidity 
after lung resection. Am Rev Respir Dis 1989; 139: 902-910.

58. Olsen GN. The evolving role of exercise testing prior to lung resection. Chest 1989; 95: 218-225.

59. Boysen PS. Perioperative management of the thoracotomy patient. Clin Chest Med 1993; 14: 321-333.

60. Bolliger CT, Wyser C, Roser H, Solèr M, Perruchoud AP. Lung scanning and exercise testing for the prediction of postoperative performance in lung resection candidates at increased risk for complications. Chest 1995; 108: 341-348.

61. American Thoracic Society: ATS Statement. Standards for the diagnosis and care of patients with chronic obstructive pulmonary disease. Am J Respir Crit Care Med 1995; 152: 77-120.

62. Sciurba FC, Rogers RM, Keenan RJ, et al. Improvement in pulmonary function and elastic recoil after lungreduction surgery for diffuse empysema. $N$ Engl J Med 1996; 334: 1095-1099.

63. American Thoracic Society. Pulmonary rehabilitation. Am Rev Respir Dis 1981; 124: 663-666.

64. Fishman AP. Pulmonary rehabilitation research. Am J Crit Care 1994; 149: 825-833.

65. Make BJ. Pulmonary rehabilitation: myth or reality. Clin Chest Med 1986; 7: 519-540.

66. Casaburi R, Petty TL. Principles and Practice of Pulmonary Rehabilitation. Philadelphia, W.B. Saunders, 1993.

67. McGavin CR, Gupta SP, Lloyd EL, et al. Physical rehabilitation for the chronic bronchitic: results of a controlled trial of exercises in the home. Thorax 1977; 32: 307-311.

68. Celli B, Rassulo J, Make BJ. Dyssynchronous breathing is associated with arm but not leg exercise in chronic obstructive pulmonary disease. N Engl J Med 1986; 314: 1485-1490.

69. Guyatt GH, Townsend M, Berman LB, Pugsely SD. Quality of life in patients with chronic airflow limitation. Br J Dis Chest 1987; 81: 45-54.

70. Atkins CJ, Kaplan RM, Timms RM, Reinsch S, Lofback $\mathrm{K}$. Behavioral exercise programs in the management of chronic obstructive pulmonary disease. J Consult Clin Physiol 1984; 52: 591-603.

71. Ries AL, Kaplan RM, Limberg TM, Prewitt LM. Effects of pulmonary rehabilitation on physiologic and psychosocial outcomes in patients with chronic obstructive pulmonary disease. Ann Intern Med 1995; 122: 823832.

72. Couser JIJ, Guthmann R, Hamadeh MA, Kane CS. Pulmonary rehabilitation improves exercise capacity in older elderly patients with COPD. Chest 1995; 107 : 730-734.

73. Casaburi R, Patessio A, Ioli F, Zanaboni S, Donner CF, Wasserman K. Reductions in exercise lactic acidosis and ventilation as a result of exercise training in patients with obstructive lung disease. Am Rev Respir Dis 1991; 143: 9-18.

74. Griffiths T, Gregory SE, Ward SA, Saunders KB, Whipp BJ. Effect of domiciliary exercise training on ventilatory and gas-exchange kinetics in patients with severe COPD. Eur Respir J 1995; 8: 502s.

75. ATS Statement. Evaluation of impairment/disability secondary to respiratory disorders. Am Rev Respir Dis 1986; 133: 1205-1209.

76. World Health Organization. International classification of impairments, disabilities, and handicaps. A manual of classification relating to the consequences of disease. Published for trial purposes in accordance with resolu- tion WHO29.35 of the Twenty-ninth World Health Assembly, May 1976. Geneva, Schuler, A.G. 1980.

77. Marshall SE, Kramer MR, Lewiston NJ, Starnes VA, Theodore J. Selection and evaluation of recipients for heart-lung and lung transplantation. Chest 1990; 98: 1488-1494.

78. Mudge GH, Goldstein S, Addonizio LJ, et al. 24th Bethesda Conference: Cardiac Transplantation. Task force 3: recipients guidelines/prioritization. $J$ Am Coll Cardiol 1993; 22: 21-31.

79. Warner Stevenson L. Role of exercise testing in the evaluation of candidates for cardiac transplantation. In: Wasserman K, ed. Exercise Gas Exchange in Heart Disease. New York, Armonk, Futura Publishing Company, 1996; Chapter 19.

80. Craven JL, Bright J, Dear CL. Psychiatric, psychosocial, and rehabilitative aspects of lung transplantation. Clin Chest Med 1990; 11: 247-257.

81. Casaburi R. Deconditioning. In: Fishman AP, ed. Pulmonary Rehabilitation. New York, Marcel Dekker, 1996; pp. 213-230.

82. The Toronto Lung Transplant Group. Experience with single-lung transplantation for pulmonary fibrosis. JAMA 1988; 259: 2258-2262.

83. Howard DK, Iademarco EJ, Trulock EP. The role of cardiopulmonary exercise testing in lung and heart-lung transplantation. Clin Chest Med 1994; 15: 405-420.

84. Levy RD, Ernst P, Levine SM, et al. Exercise performance after lung transplantation. J Heart Lung Transplant 1993; 12: 27-33.

85. Roca J, Whipp BJ, eds. Clinical Exercise Testing. Eur Respir Mon 1997; (in press).

86. Kavanagh T, Yacoub MH, Mertens DJ, Kennedy J, Campbell RB, Sawyer P. Cardiorespiratory responses to exercise training after orthotopic cardiac transplantation. Circulation 1988; 77: 162-171.

87. Jones NL. Clinical exercise testing. Philadelphia, W.B. Saunders, 1980.

88. Whipp BJ, Davis JA, Torres F, Wasserman K. A test to determine parameters of aerobic function during exercise. J Appl Physiol 1981; 50: 217-221.

89. Sterk PJ, Fabbri LM, Quanjer PH, et al. Airway responsiveness. Standardized challenge testing with pharmacological physical and sensitizing stimuli in adults. Eur Respir J 1993; 6: 53-83.

90. Balke B, Ware RW. An experimental study of "physical fitness" of Air Force personnel. US Armed Forces Med J 1959; (Abstract).

91. Zeballos RJ, Weisman IM. Behind the scenes of cardiopulmonary exercise testing. Clin Chest Med 1994; 15: 193-213.

92. Mador MJ, Rodis A, Magalang UJ. Reproducibility of borg scale measurements of dyspnea during exercise in patients with COPD. Chest 1995; 107: 1590-1597.

93. Hansen JE. Participant responses to blood gas proficiency testing reports. Chest 1992; 101: 1240-1244.

94. Clark JS, Votteri B, Arriagno RL, et al. Noninvasive assessment of blood gases. Am Rev Respir Dis 1992; 145: 220-232.

95. Hughes JMB. Blood gas estimations from arterialized capillary blood versus arterial puncture: are they different? Eur Respir J 1996; 9: 184-185.

96. Sauty A, Uldry C, Debétaz L, Leuenberger P, Fitting J. Differences in $\mathrm{PO}_{2}$ and $\mathrm{PO}_{2}$ between arterial and arterialized earlobe samples. Eur Respir J 1996; 9: 186-189.

97. Hughson RL, Weisiger KH, Swanson GD. Blood lactate concentration increases as a continuous function in 
progressive exercise. J Appl Physiol 1987; 62: 1975 1981.

98. Anderson SD. Exercise-induced asthma. In: O'Byrne P, Thompson NC, eds. Manual of Asthma Management. London, W.B. Saunders Co., 1995; pp. 621-643.

99. Cropp GJA. The exercise bronchoprovocation test: standardization of procedures and evaluation of response. $J$ Allergy Clin Immunol 1979; 64: 627-633.

100. Haby MM, Peat JK, Mellis CM, Anderson SD, Woolcock AJ. An exercise challenge for epidemiological studies of childhood asthma: validity and repeatability. Eur Respir J 1995; 8: 729-736.

101. Strauss RE, McFadden ER, Ingram RH, Deal EC, Jaeger JJ. Influence of heat and humidity on the airway obstruction induced by exercise in asthma. $J$ Clin Invest 1978; 61: 433-440.

102. McFadden ER, Lenner KAM, Kingman PS. Post exertional airway rewarming and thermally induced asthma: new insights and pathophysiology and possible pathogenesis. J Clin Invest 1986; 78: 18-25.

103. Anderson SD, Schoeffel RE, Follet R, Perry CP, Daviskas E, Kendall M. Sensitivity to heat and water loss at rest and during exercise in asthmatic patients. Eur J Respir Dis 1982; 63: 459-471.

104. Anderson SD, Daviskas E. The airway microvasculature and exercise induced asthma. Thorax 1992; 47: $748-752$.

105. Anderson SD, Silverman M, Konig F, Godfrey S. Exercise-induced asthma. Brit J Dis Chest 1975; 69: 1-39.

106. Givoni B, Goldman RF. Predicting metabolic energy cost. J Appl Physiol 1971; 30: 429-433.

107. Silverman M, Anderson SD. Metabolic cost of treadmill exercise in children. J Appl Physiol 1972; 33: 696698.

108. Noviski N, Bar-Yishay E, Gur I, Godfrey S. Exercise intensity determines and climatic conditions modify the severity of exercise-induced asthma. Am Rev Respir Dis 1987; 136: 592-594.

109. Phillips YY, Jaeger JJ, Laube BL, Rosenthal RR. Eucapnic voluntary hyperventilation of compressed gas mixture: a simple system for voluntary hyperventilation of compressed gas mixture: a simple system for bronchial challenge by respiratory heat loss. Am Rev Respir Dis 1985; 131: 31-35.

110. Rodwell LT, Anderson SD, Du Toit J, Seale JP. Different effects of inhaled amiloride and furosemide on airway responsiveness to dry air challenge in asthmatic subjects. Eur Respir J 1993; 6: 855-861.

111. Anderson SD, Rodwell LT, Du Toit J, Young IH Duration of protection of inhaled salmeterol in exercise-induced asthma. Chest 1991; 100: 1254-1260.

112. Woolley M, Anderson SD, Quigley B. Duration of protective effect of terbutaline sulphate and cromolyn sodium alone and in combination on exercise-induced asthma. Chest 1990; 97: 39-45.

113. Kemp JP, Dockhorn RJ, Busse WW, Blaecker NR, Van As A. Prolonged effect of inhaled salmeterol against exercise-induced bronchospasm. Am J Respir Crit Care Med 1994; 150: 1612-1615.

114. Bruce RA. Exercise testing of patients with coronary artery disease. Ann Clin Res 1971; 3: 323-332.

115. Patterson JA, Naughton J, Pietras RJ, Gumar RN. Treadmill exercise in assessment of patients with cardiac disease. Am J Cardiol 1972; 30: 757-762.

116. Cotes JE. Lung function: Assessment and Application in Medicine. Fifth edition. London, Blackwell Scientific Publishers 1993.
117. Buchfuhrer MJ, Hansen JE, Robinson TE, Sue DY, Wasserman K, Whipp BJ. Optimizing the exercise protocol for cardiopulmonary assessment (Abstract). J Appl Physiol 1983; 55: 1558-1564.

118. Astrand PO, Rodahl K. Textbook of Work Physiology. New York, McGraw-Hill, 1986.

119. Hermansen L, Saltin B. Oxygen uptake during maximal treadmill and bicycle exercise. J Appl Physiol 1969; 26: 1-31.

120. McArdle WD, Katch RI, Pechar GS. Comparison of continuous and discontinuous treadmill and bicycle tests for max $V^{\prime} \mathrm{O}_{2}$. Med Sci Sports Exerc 1973; 5: 156-160.

121. McKay GA, Banister EW. A comparison of maximum oxygen uptake determination of bicycle ergometry at various pedaling frequencies and by treadmill running at various speeds. Eur J Appl Physiol 1976; 35: 191200.

122. Koyal SN, Whipp BJ, Huntsman D, Bray GA, Wasserman $\mathrm{K}$. Ventilatory responses to the metabolic acidosis of treadmill and cycle ergometry. J Appl Physiol 1976; 40: 864-867.

123. Whipp BJ. The Physiological and Energetic Basis of Work Efficiency. Bray GA, ed. Obesity in Perspective. Washington DC, U.S. Govt. Printing Office, 1976; pp. 121-126.

124. Whipp BJ, Bray GA, Koyal SN, Wasserman K. Exercise Energetics and Respiratory Control in Man Following Acute and Chronic Elevation of Calorie Intake. Obesity in Perspective. Bray GA, ed. Washington DC, U.S. Govt. Printing Office, 1976; pp. 157-163.

125. Wasserman K, Whipp BJ. Exercise physiology in hea1th and disease (state of the Art). Am Rev Respir Dis 1975; 112: 219-249.

126. Lanooy C, Bonjer FH. A hyperbolic ergometer for cycling and cranking. J Appl Physiol 1956; 9: 499-500.

127. American Heart Association Subcommittee on Rehabilitation Target Activity Group, Hellerstein HKC. Specification for exercise testing equipment. Circulation 1979; 59: 849A-854A

128. Davis JA, Whipp BJ, Lamarra N, Huntsman DJ, Frank $\mathrm{MH}$, Wasserman K. Effect of ramp slope on measurement of aerobic parameters from the ramp exercise test. Med Sci Sports Exerc 1982; 14: 339-343.

129. Hansen JE, Casaburi R, Cooper DM, Wasserman K. Oxygen uptake as related to work rate increment during cycle ergometer exercise. Eur J Appl Physiol 1988; 57: 140-145.

130. Zhang YY, Johnson MC, Chow N, Wasserman K. Effect of exercise testing protocol on parameters of aerobic function. Med Sci Sports Exerc 1991; 23: 625-630.

131. Myers J, Buchanan N, Walsh D, et al. Comparison of the ramp versus standard exercise protocols. J Am Coll Cardiol 1991; 17: 1334-1342.

132. Tanner CS, Heise CT, Barber G. Correlation of the physiologic parameters of a continuous ramp versus an incremental James exercise protocol in normal children. Am J Cardiol 1991; 67: 309-312.

133. Naughton J. Exercise Testing. Physiological, Biochemical and Clinical Principles. New York, Futura Publishing Company, 1988

134. Beaver WL. Water vapour corrections in oxygen consumption calculations. J Appl Physiol 1973; 35: 928 931.

135. Poole GW, Maskell RC. Validation of continuous determination of respired gases during steady-state exercise. J Appl Physiol 1975; 38: 736-738.

136. Spiro SG, Juniper E, Bowman P, Edwards RHT. An 
increasing work rate test for assessing the physiological strain of submaximal exercise. Clin Sci Mol Med 1974; 46: 191-206.

137. Beaver WL, Wasserman K, Whipp BJ. On-line computer analysis and breath-by-breath graphical display of exercise function tests. J Appl Physiol 1973; 34: 128132.

138. Noguchi H, Ogushi Y, Yoshiya N, Itakura N, Yamabayashi $\mathrm{H}$. Breath-by-breath $V \mathrm{CO}_{2}$ and $V_{\mathrm{O}_{2}}$ require compensation for transport delay and dynamic response. $J \mathrm{Appl}$ Physiol 1982; 52: 79-84.

139. Yamamoto Y, Takei Y, Mokushi K, Morita H, Mutoh Y, Miyashita M. Breath-by-breath measurement of alveolar gas exchange with a slow-response gas analyser. Med Biol Eng Comput 1987; 25: 141-146.

140. Beaver WL, Lamarra N, Wasserman K. Breath-by-breath measurement of true alveolar gas exchange. J Appl Physiol 1981; 51: 1662-1675.

141. Sue DY, Hansen JE, Blias M, Wasserman K. Measurement and analysis of gas exchange during exercise using a programmable calculator. J Appl Physiol 1980; 49: 456-461.

142. Lamarra N, Whipp BJ. Measurement of pulmonary gas exchange. In: Maud PJ, Foster C, eds. Physiological Assessment of Human Fitness. Champaign, IL, USA, Human Kinetics, 1995; pp. 19-35.

143. Wilmore JH, Costill DL. Adequacy of the Haldane transformation in the computation of exercise $V^{\prime} \mathrm{O}_{2}$ in man. J Appl Physiol 1973; 35: 85-98.

144. Swanson GD. Breath-by-breath considerations for gas exchange kinetics. In: Ceretelli P, Whipp BJ, eds. Exercise Bioenergetics and Gas Exchange. Amsterdam, Elsevier/ North Holland, 1980; pp. 211-222.

145. Dennis SC, Noakes TD, Bosch AN. Ventilation and blood lactate increase exponentially during incremental exercise. J Sports Sci 1992; 10: 437-449.

146. Wasserman K, Beaver WL, Whipp BJ. Mechanisms and pattern of blood lactate increase during exercise in man Med Sci Sports Exerc 1986; 18: 344-352.

147. Beaver WL, Wasserman K, Whipp BJ. Bicarbonate buffering of lactic acid generated during exercise. J Appl Physiol 1986; 60: 472-478.

148. Clausen JL. Pulmonary Function Testing. Guidelines and Controversies. Equipment, Methods and Normal Values. Orlando, USA, Grune \& Stratton, Inc. 1984; pp. 1-338.

149. Quanjer PH, Tammeling GJ, Cotes JE, Pedersen OF, Peslin R, Yernault JC. Lung volumes and forced ventilatory flows. Report Working Party: standardization of lung function testing. Eur Respir J 1993; 6: 5-40.

150. American Thoracic Society. Standardization of spirometry: 1994 update. Am J Respir Crit Care Med 1995; 152: $1107-1136$.

151. Finucane KE, Egan BA, Dawson SV. Linearity and frequency response of pneumotachographs. J Appl Physiol 1972; 32: 121-126.

152. Miller MR, Pincock AC. Linearity and temperature control of the Fleisch pneumotachograph. J Appl Physiol 1986; 60: 710-715

153. Porszasz J, Barstow TJ, Wasserman L. Evaluation of a symmetrically disposed Pitot tube flowmeter for measuring gas flow during exercise. J Appl Physiol 1994; 77: 2659-2665.

154. Yoshiya I, Nakajima T, Nagai I, Jitsukawa S. A bidirectional respiratory flowmeter using the hot-wire principle. J Appl Physiol 1975; 38: 360-365.

155. Yoshiya I, Shimada Y, Tanaka K. Evaluation of a hot- wire respiratory flowmeter for clinical applicability. $J$ Appl Physiol 1979; 47: 1131-1135.

156. Yeh MP, Adams TD, Gardner RM, Yanowitz FG. Turbine flow meter $v s$ Fleisch pneumotachometer: a comparative study for exercise testing. J Appl Physiol 1987; 63: 1289-1295.

157. Cooper CB, Harris ND, Howard P. Evaluation of a turbine flow meter (Ventilometer Mark 2) in the measurement of ventilation. Respiration 1990; 57: 243-247.

158. Mogue LR, Rantala B. Capnometers. J Clin Monitoring 1988; 4: 115-121.

159. Fletcher GF, Froelicher VF, Hartley LH, Haskell WL, Pollack ML. Exercise standards: a statement for health professionals from the American Heart Association. Circulation 1990; 82: 2286-2322.

160. American Heart Association. Exercise standards. Circulation 1995; 91: 580-615.

161. Council on Scientific Affairs American Medical Association. The use of pulse oximetry during conscious sedation. JAMA 1993; 270: 1463-1468.

162. Tobin MJ. Respiratory monitoring. JAMA 1990; 264: 244-251.

163. Ries AL, Farrow JT, Clausen JL. Accuracy of two ear oximeters at rest and during exercise in pulmonary patients. Am Rev Respir Dis 1985; 132: 685-689.

164. Carlin BW, Clausen JL, Ries AL. The use of cutaneous oximetry in the prescription of long-term oxygen therapy. Chest 1988; 94: 239-241.

165. Orenstein DM, Curtis SE, Nixon PA, Hartigan ER. Accuracy of three pulse oximeters during exercise and hypoxemia in patients with cystic fibrosis. Chest 1993; 104(4): 1187-1190.

166. Carlin BW, Clausen JL, Ries AL. The effects of exercise testing on the prescription of oxygen therapy. Chest 1994; 106: 361-365.

167. Hansen JE, Casaburi R. Validity of ear oximetry in clinical exercise testing. Chest 1987; 91: 333-337.

168. Ries AL, Prewitt LM, Johnson JJ. Skin color and ear oximetry. Chest 1989; 96: 287-290.

169. Zeballos RJ, Weisman IM. Reliability of noninvasive oximetry in black subjects during exercise and hypoxia. Am Rev Respir Dis 1991; 144: 1240-1244.

170. Ramsey M. Blood pressure monitoring: automated oscillometric devices. J Clin Monitoring 1991; 7: 56-57.

171. Robinson TR, Sue DY, Huszczuk A, Weiler-Ravell D, Hansen JE. Intra-arterial and cuff blood pressure responses during incremental cycle ergometry. Med Sci Sports Exerc 1988; 20: 142-149.

172. Consolazio CF, Johnson RE, Pecora LJ. Physiological measurements of metabolic functions in man. New York, McGraw-Hill, 1963.

173. Huszczuk A, Whipp BJ, Wasserman K. A respiratory gas exchange simulator for routine calibration in metabolic studies. Eur Respir J 1990; 3: 465-468.

174. Clark JH, Greenleaf JE. Electronic bicycle ergometer: a simple calibration procedure. J Appl Physiol 1971; 30: 440-442.

175. Van Praagh E, Bedu M, Roddier P, Coudert J. A simple calibration method for mechanically braked cycle ergometers. Int J Sports Med 1992; 13: 27-30.

176. Russell JC, Dale JD. Dynamic torquemeter calibration of bicycle ergometers. J Appl Physiol 1986; 61: 1217-1220.

177. Gardner RM, Schwartz R, Wong HC, et al. Percutaneous indwelling radial-artery catheters for monitoring cardiovascular function. N Engl J Med 1974; 290: 12271231.

178. Douard H, Mora B, Broustet JP. Epreuves d'effort et 
tachycardies ventriculaires: l'expérience française. Arch Mal Coeur 1987; 80: 263-270.

179. Simel DL, Samsa GP, Matcha DB. Likelihood ratios with confidence: sample size estimation for diagnostic test studies. J Clin Epidemiol 1991; 44: 763-770.

180. Evidence-Based Medicine Working Group. Evidencebased medicine: a new approach to the teaching of medicine. JAMA 1992; 268: 2420-2425.

181. Jones NL, Makrides L, Hitchcock C, Chypchar T, McCartney N. Normal standards for an incremental progressive cycle ergometer test. Am Rev Respir Dis 1985; 131: 700-708.

182. Jones NL, Summers E, Killian KJ. Influence of age and stature on exercise capacity during incremental cycle ergometry in men and women. Am Rev Respir Dis 1989; 140: $1373-1380$.

183. Blackie SP, Fairbarn MS, McElvaney NG, Wilcox PG, Morrison NJ, Pardy RL. Normal values and ranges for ventilation and breathing pattern at maximal exercise. Chest 1991; 100: 136-142.

184. Blackie SP, Fairbarn MS, McElvaney NG, Morrison NJ, Wilcox PG, Pardy RL. Prediction of maximal oxygen update and power during cycle ergometry in subjects older than 55 years of age. Am Rev Respir Dis 1989; 139: 1424-1429.

185. Fairbarn MS, Blackie SP, McElvaney NG, Wiggs BR, Paré PD, Pardy RL. Prediction of heart rate and oxygen uptake during incremental and maximal exercise in healthy adults. Chest 1994; 105: 1365-1369.

186. Bruce RA, Kusumi MS, Hosmer D. Maximal oxygen intake and nomographic assessment of functional aerobic impairment in cardiovascular disease. Am Heart $J$ 1973; 85: 546-562.

187. Vogel JA, Patton JF, Mello RP, Daniels WL. An ana- lysis of aerobic capacity in a large United States population. J Appl Physiol 1986; 60: 494-500.

188. Pollock ML, Bohannon RL, Cooper KHE. A comparative analysis of four protocols for maximal treadmill stress testing. Am Heart J 1976; 92: 39-46.

189. Shephard RJ, Allen C, Benade AJ. The maximal oxygen intake: an international reference standard of cardiorespiratory fitness. Bull World Health Organization 1968; 38: 757-764.

190. Taylor HL, Buskirk E, Henschel A. Maximal oxygen intake as an objective measure of cardio-respiratory performance. J Appl Physiol 1955; 8: 73-80.

191. Wagner PD, Gale GE, Moon RE, Torre-Bueno JE, Stolp BW, Saltzman HA. Pulmonary gas exchange in humans exercising at sea level and simulated altitude. J Appl Physiol 1986; 61: 260-270.

192. Young DS. Implementation of SI units for clinical laboratory data. Style specifications and conversion tables. Ann Intern Med 1987; 106: 114-129.

193. Cotes JE. SI units in respiratory medicine. Am Rev Respir Med 1975; 112: 753-755.

194. Ward SA, Whipp BJ. Influence of body $\mathrm{CO}_{2}$ stores on ventilatory-metabolic coupling during exercise. In: Honda Y, Miyamoto K, Konno K, Widdicombe JG, eds. Control of Breathing and its Modeling Perspective. New York, Plenum Press, 1992; pp. 425-431.

195. Beaver WL, Wasserman K, Whipp BJ. A new method for detecting the anaerobic threshold by gas exchange. J Appl Physiol 1986; 60: 2020-2027.

196. Sue DY, Wasserman K, Moricca RB, Casaburi R. Metabolic acidosis during exercise in patients with chronic obstructive pulmonary disease. Use of v-slope method for anaerobic threshold determination. Chest 1988; 94 : 931-938. 